\title{
Multiscale High-order/Low-order (HOLO) Algorithms and Applications
}

\author{
L. Chacón ${ }^{\mathrm{a}, *}$, G. Chen ${ }^{\mathrm{a}}$, D. A. Knoll ${ }^{\mathrm{a}}$, C. Newman ${ }^{\mathrm{a}}$, H. Park $^{\mathrm{a}}$, W. Taitano ${ }^{\mathrm{a}}$, J. A. Willert ${ }^{\mathrm{b}}$, G. Womeldorff ${ }^{\mathrm{a}}$ \\ ${ }^{a}$ Los Alamos National Laboratory, Los Alamos, NM 87545 \\ ${ }^{b}$ Institute for Defense Analyses, Alexandria, VA 22311
}

\begin{abstract}
We review the state of the art in the formulation, implementation, and performance of so-called highorder/low-order (HOLO) algorithms for challenging multiscale problems. HOLO algorithms attempt to couple one or several high-complexity physical models (the high-order model, HO) with low-complexity ones (the low-order model, LO). The primary goal of HOLO algorithms is to achieve nonlinear convergence between $\mathrm{HO}$ and $\mathrm{LO}$ components while minimizing memory footprint and managing the computational complexity in a practical manner. Key to the HOLO approach is the use of the LO representations to address temporal stiffness, effectively accelerating the convergence of the $\mathrm{HO} / \mathrm{LO}$ coupled system. The HOLO approach is broadly underpinned by the concept of nonlinear elimination, which enables segregation of the $\mathrm{HO}$ and LO components in ways that can effectively use heterogeneous architectures. The accuracy and efficiency benefits of HOLO algorithms are demonstrated with specific applications to radiation transport, gas dynamics, plasmas (both Eulerian and Lagrangian formulations), and ocean modeling. Across this broad application spectrum, HOLO algorithms achieve significant accuracy improvements at a fraction of the cost compared to conventional approaches. It follows that HOLO algorithms hold significant potential for high-fidelity system scale multiscale simulations leveraging exascale computing.
\end{abstract}

Keywords: high-order/low-order, HOLO, multiscale algorithms, implicit timestepping, communication avoiding algorithms

\section{Contents}

1 Introduction $\quad 2$

2 HOLO algorithms, complexity reduction, and nonlinear elimination 4

3 Application 1: Radiation transport $\quad 5$

3.1 Radiation transport problem formulation $\ldots \ldots \ldots \ldots \ldots$

3.2 Thermal radiative transfer equation $\ldots \ldots \ldots \ldots \ldots \ldots \ldots$

3.3 Numerical Examples . . . . . . . . . . . . . . . . . . . . . . . . . 8

3.3.1 1D gray Marshak wave problem $\ldots \ldots \ldots \ldots \ldots \ldots \ldots \ldots$

*Corresponding author

Email address: chacon@lanl.gov (L. Chacón)

(C) 2016. This manuscript version is made available under the Elsevier user license http://www.elsevier.com/open-access/userlicense/1.0/ 
3.3.2 Monte Carlo HO solver extension $\ldots \ldots \ldots \ldots \ldots \ldots$

3.3.3 Residual Monte Carlo HO solver . . . . . . . . . . . . . . . . . . . . . . . . . . 10

4 Application 2: Gas dynamics $\quad 11$

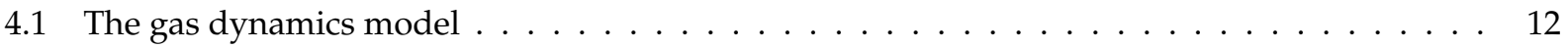

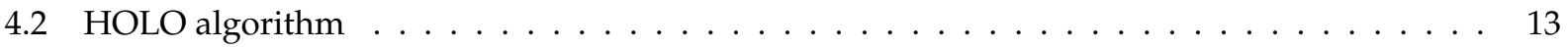

4.3 Numerical results . . . . . . . . . . . . . . . . . . . . . . . . . . 15

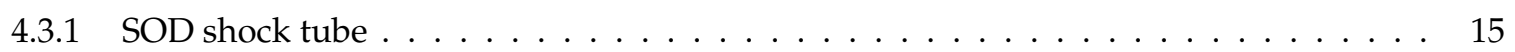

4.3.2 Strong shock tube with variable $\tau \ldots \ldots \ldots \ldots \ldots \ldots$

5 Application 3: Plasma kinetic HOLO algorithms $\quad 18$

5.1 Collisionless Particle-in-cell HOLO algorithms $\ldots \ldots \ldots \ldots \ldots \ldots$

5.1 .1 The Vlasov-Darwin model . . . . . . . . . . . . . . . . . . . . . . . . . . . 19

5.1 .2 The HOLO PIC algorithm . . . . . . . . . . . . . . . . . . . . . 19

5.1 .3 Numerical results . . . . . . . . . . . . . . . . . . . . . . . . . . . . . . 20

5.2 Collisional Vlasov-Fokker-Planck HOLO algorithms . . . . . . . . . . . . . . . . 23

5.2.1 The Vlasov-Fokker-Planck model . . . . . . . . . . . . . . . . . . . . . . . . 24

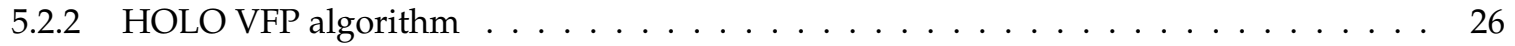

5.2 .3 Numerical results . . . . . . . . . . . . . . . . . . . . . . . . . . 27

6 Application 4: Ocean modeling 29

$6.1 z$-level ocean model . . . . . . . . . . . . . . . . . . . . . . . . . . . . . . 30

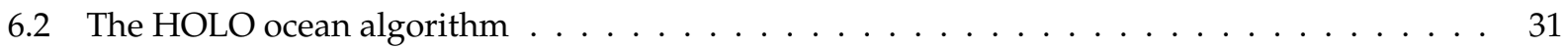

6.3 Numerical results . . . . . . . . . . . . . . . . . . . . . 32

7 Conclusions $\quad 34$

\section{Introduction}

The advent of exascale computing promises to revolutionize multiscale simulation capabilities. The sheer availability of cycles, combined with hierarchical architectures, may allow targeted deployment of algorithms to architectures that are best suited for them. Whether this promise comes to pass, however, will depend critically on the availability of suitable algorithmic strategies. These algorithms will need to be able to bridge the scale gap temporally and spatially, and will have to provide an unprecedented level of fidelity while delivering a manageable computational complexity and optimal scalability (not only in parallel, but algorithmically as well, i.e., providing computational complexities that scale linearly with the number of degrees of freedom).

This review paper focuses on recent developments in a novel type of multiscale algorithm, suitable for problems in which a microscopic description needs to be coupled to a macroscopic one. Such descriptions arise in many application domains where multiphysics simulations are needed. Microscopic descrip- 
tions typically arise from some high-dimensional formulation of the problem (e.g, Boltzmann equation in fluids and plasmas, molecular dynamics simulations, etc.), while macroscopic descriptions typically describe some mean-field behavior of the problem, representing either independent physics (e.g., Maxwell's equations), or obtained by some coarse-graining procedure from the microscopic description (e.g., moment coarsening). For the purposes of this paper, we will term the microscopic system the high-order (HO) description, and the macroscopic one the low-order (LO) description, and the resulting algorithm will be termed HOLO.

HOLO-type algorithms are not strictly new, with history going back to the 60s, 80s, and 90s in disciplines such as radiation transport [1, 2, 3, 4], plasma physics [5, 6, 7, 8], and ocean modeling [9]. However, with the advent of modern nonlinear solver technology and faster computers, algorithmic solutions that were unthinkable a few decades ago have become tractable. Summarizing the state of the art in HOLO multiscale algorithmic solutions is the principal focus of this review paper. In particular, a common feature in many such HOLO formulations is the need for tight nonlinear coupling, owing to the fact that the coupling is bidirectional: LO physics drives the HO dynamics (e.g. temperature determines photon absorption/reemission; electromagnetic fields determine particle orbits), and HO quantities inform LO dynamics (e.g., kinetic closures). In such instances, operator-splitting approaches fail to capture the correct behavior unless the fastest time scales in either $\mathrm{HO}$ or LO descriptions are resolved, and therefore do not provide a suitable multiscale strategy. It follows that implicit time stepping, perhaps in combination with multirate/IMEX strategies, and the associated iterative nonlinear solver technology are needed for a truly multiscale integration of these equations. This, in turn, brings the high dimensionality of the HO system to the fore, as a naive formulation, with both $\mathrm{HO}$ and $\mathrm{LO}$ degrees of freedom represented explicitly in the nonlinear residual, would quickly become impractical even in the largest parallel computers.

One key modern approach to deal with the complexity challenge of $\mathrm{HO}$ models is nonlinear elimination [10], in which the HO component is enslaved to the LO one. With nonlinear elimination, HO degrees of freedom are no longer considered dependent variables, but are actually implemented as auxiliary computations in the evaluation of a nonlinear residual. Nonlinear elimination reduces complexity since the $\mathrm{HO}$ system becomes invisible to the nonlinear solver, greatly reducing its memory footprint and overhead. The price to pay is that the evaluation of the nonlinear residual itself remains expensive. As a consequence, minimizing the number of residual evaluations is paramount, which in turn shifts the discussion towards effective convergence acceleration strategies, e.g. preconditioning.

Indeed, a key component of the HOLO algorithm is the acceleration of the LO system solve. Only the LO system needs to be considered owing to the elimination of $\mathrm{HO}$ variables from the residual. This is a key advantage of the HOLO approach, as typically there is a wealth of experience dealing with such LO solvers in various communities that can be leveraged for the development of suitable preconditioning strategies. This will be a recurrent theme in the sections that follow in this paper.

A key aspect, also a recurrent theme, is the need for discrete consistency between $\mathrm{HO}$ and $\mathrm{LO}$ formulations 
for long-term accuracy and nonlinear stability. It has been observed by many HOLO practitioners that disregarding this requirement leads to unworkable algorithms. There are several strategies to deal with this requirement. One is to reset LO quantities with HO-computed ones at the beginning of the time step [11, 12], but this does not enforce true consistency [13]. Another one is to introduce so-called consistency terms, to enforce that $\mathrm{LO}$ solutions exactly match $\mathrm{HO}$ ones in the limit of arbitrarily small nonlinear convergence tolerances [14, 15, 16, 13, 17]. A third strategy is to consider a minimalistic HOLO formulation in the nonlinear residual, which avoids discrete consistency issues, and use an augmented LO model only to compute linear responses in the preconditioner stage $[18,19,20]$. We will show examples of all these in the sections that follow.

The rest of the manuscript is organized as follows. Section 2 will introduce in detail the complexity reduction strategy employed in HOLO algorithms. Sections 3-6 will introduce various applications of the HOLO algorithmic strategy, including thermal radiation transport (Sec. 3), gas dynamics (Sec. 4), plasmas (Sec. 5, including both Lagrangian -Sec. 5.1- and Eulerian -Sec 5.2- models), and ocean modeling (Sec. 6). We conclude in Sec. 7.

\section{HOLO algorithms, complexity reduction, and nonlinear elimination}

The starting point of the HOLO algorithm is one or more high-order (HO), high-complexity descriptions (e.g., a microscopic model such as kinetic formulation in phase space), nonlinearly coupled to one or more low-order (LO) descriptions of lower complexity (e.g., a macroscopic description such as moment equations). The case of interest is when the nonlinear coupling between these descriptions is tight, i.e., a scale separation argument cannot be used to treat these descriptions semi-independently (for instance, by operator splitting).

In such cases, a nonlinear solution is required that iterates these $\mathrm{HO}$ and $\mathrm{LO}$ systems to convergence. This nonlinearly coupled set of equations can be conceptually formulated as a nonlinear residual of the form:

$$
\mathbf{F}(\mathbf{X}, \boldsymbol{\xi})=0
$$

Here, $\mathbf{X}$ represents the low-order (macro) model, and $\xi$ the high-order (micro) one. In formulating this residual, we have naively taken both macro and micro variables as dependent variables. While in principle correct, this formulation has one fundamental limitation, namely, that the residual has inherited the full dimensionality of the microscopic problem. For any iterative solution method employing the residual in Eq. 1 , this will result in exceedingly large storage requirements. Given that memory is the principal bottleneck in current and future massively parallel computers, pursuing the formulation in Eq.(1) does not appear promising.

There is, however, an alternative, which is to nonlinearly enslave the $\mathrm{HO}$ component to the $\mathrm{LO}$ one. The concept of nonlinear enslavement (or elimination [10]) is akin to the well-known elimination process in linear equations, and can be explained as follows. Let us consider the global nonlinear residual $\mathbf{F}(\mathbf{X}, \boldsymbol{\xi})$, 
and decompose it into two sets of equations, $\mathbf{F}_{H O}(\mathbf{X}, \boldsymbol{\xi})=0$ and $\mathbf{F}_{L O}(\mathbf{X}, \boldsymbol{\xi})=0$, and assume that $\mathbf{F}_{H O}(\mathbf{X}, \boldsymbol{\xi})$ can be straightforwardly written (albeit still with high complexity) in an explicit form as $\xi=\mathbf{f}_{H O}(\mathbf{X})$. It follows that a new residual can be formulated as:

$$
\mathbf{F}_{L O}\left(\mathbf{X}, \mathbf{f}_{H O}(\mathbf{X})\right)=\mathbf{G}_{L O}(\mathbf{X})=0
$$

By construction, the new residual $\mathbf{G}_{L O}$ has much lower dimensionality than the original one $\mathbf{F}$ (and thus requires less storage for the Krylov and Newton iterations), but has the same nonlinear solution.

This is the essence of a HOLO algorithm: high-order quantities do not appear explicitly in the residual, and the iteration is driven by the LO model. As a result, nonlinear solver memory requirements (e.g., nonlinear residuals, Krylov subspace vectors, etc.) are comparable to those of a moment/fluid description. Only one copy of the HO solution is needed in practice. The enslavement of the HO component allows complete flexibility in the formulation of the $\xi=\mathbf{f}_{H O}(\mathbf{X})$ map. Also, since this operation is segregated in the calculation of the residual $\mathbf{G}_{L O}$, it is ideally suited to exploit heterogeneous computing architectures. Finally, by adequately formulating the LO model such that stiff time scales are represented, one can very effectively accelerate the nonlinear iteration just by aggressively attacking numerical stiffness of the LO model, e.g. via preconditioning. This enables the use of suitable $\mathrm{LO}$ formulations to accelerate the nonlinear convergence of the HOLO algorithm.

We consider next a few applications that exemplify the power of the HOLO algorithm. The choice of nonlinear iterative method is flexible, and problem-dependent. In the applications that follow, we have explored the use of simple fixed-point (Picard) algorithms, Jacobian-free Newton-Krylov (JFNK [21, 22]), as well as accelerated fixed-point methods (e.g., Anderson acceleration [23, 24]).

\section{Application 1: Radiation transport}

Accurate modeling of neutral particle transport behavior is of great importance in many science and engineering applications $[25,26]$. In general, neutral particle transport phenomena is described by the Boltzmann transport equation. Numerical algorithms for solving the transport equation often suffer from slow convergence of stiff collision-like physics, such as scattering, fission and/or absorption-reemission. Moment-based scale-bridging algorithms [15, 16, 27, 28, 29, 30, 31, 32, 33] have shown great potential for accelerating the solution of the Boltzmann transport equation by bridging the diffusion and transport scales. The basic idea behind the moment-based scale-bridging algorithm is to utilize low-order (LO) continuum equations that are consistently derived from the high-order $(\mathrm{HO})$ transport equation. Due to discrete consistency, the LO system can be used not only for accelerating the original transport equation, but also used for coupling to other physics.

Historically, moment-based acceleration has been very successful for solving transport equations. One popular approach is the Quasi-diffusion (QD) method [1, 34, 35, 36, 37, 38, 39], where the angularly integrated balance equation is nonlinearly closed by the second moment of the radiation field via the Eddington 
tensor. In the QD method, $\mathrm{HO}$ and LO equations are often discretized independently, although it is possible to discretize the QD equations in the algebraically consistent way. When the equations are independently discretized, the QD method converges to the original transport solution as the mesh is refined.

Another popular class of HOLO acceleration methods include nonlinear diffusion acceleration (NDA) and coarse-mesh finite difference (CMFD) [2, 40, 41, 42, 14, 15, 43, 44, 45, 33]. Rather than deriving a closure relation from the transport equation directly, NDA and CMFD utilize a modified diffusion closure by adding a discrete correction term. Unlike QD, NDA and CMFD are derived from the discrete equation. Therefore, the $\mathrm{LO}$ and $\mathrm{HO}$ solutions produce the same local conservation properties upon convergence of the nonlinear closure term, regardless of the spatial mesh size.

The diffusion synthetic acceleration (DSA), transport synthetic acceleration (TSA), and Linear Multifrequency Gray (LMFG) acceleration methods [4, 46, 47, 48, 49, 50] are also popular schemes. Unlike the moment-based acceleration method, the LO problem of DSA and TSA is linear. However, a consistent implementation of DSA can be complex, and TSA still requires the solution of a (simpler) transport problem.

\subsection{Radiation transport problem formulation}

We describe the moment-based acceleration using the following general form of (steady-state) neutral particle transport equation,

$$
\mathbf{\Omega} \cdot \nabla \psi+\sigma_{t} \psi=q,
$$

where $\psi=\psi(\mathbf{r}, \Omega, E)$ is the angular flux at the location $\mathbf{r}$, traveling in the direction of $\Omega$ with energy $E$. In thermal radiative transfer, photon frequency $v$ is commonly used instead of the energy variable $E$. The right-hand term in Eq. 3 is asource term. For neutron transport eigenvalue problems, the source term, $q$, consists of scattering and fission term,

$$
\begin{aligned}
q & =\iint \sigma_{s}\left(\mathbf{\Omega}^{\prime} \cdot \mathbf{\Omega}, E^{\prime} \rightarrow E\right) \psi\left(\mathbf{\Omega}^{\prime}, E^{\prime}\right) d E^{\prime} d \Omega^{\prime} \\
& +\frac{\chi(E)}{4 \pi k_{e f f}} \iint \bar{v} \sigma_{f}\left(E^{\prime}\right) \psi\left(\mathbf{\Omega}^{\prime}, E^{\prime}\right) d E^{\prime} d \Omega^{\prime}
\end{aligned}
$$

While for thermal radiative transfer problems, the source term becomes a black-body reemission,

$$
q=\sigma B(v, T)=\sigma \frac{2 h v^{3}}{c^{3}\left(e^{h v / k T}-1\right)}
$$

Eq. 3 is amenable to solution using the Picard iteration,

$$
\psi^{k+1}=\mathcal{G}\left(\psi^{k}\right)=\mathcal{T}^{-1} q\left(\psi^{k}\right)
$$

where $\mathcal{G}$ is a fixed-point operator. Convergence of the fixed-point iteration can be very slow when a stiff source term exists. In neutral particle transport algorithm, the operator $\mathcal{T} \equiv \Omega \cdot \nabla+\sigma$ is called the transport operator. Most of the numerical algorithms involve inversion of the transport operator and this process is referred to as a "transport sweep" in the radiation transport community. The transport sweep is a noniterative procedure, but often dominates the computational time. When transport sweeps are employed 
during Picard iteration, the iterative procedure is often referred to as source iteration. One goal of momentbased acceleration is to minimize the required number of transport sweeps by accelerating the convergence of the stiff coupling between radiation and the source term.

In the next section, we discuss in more detail the LO system formulation for the thermal radiative transfer application. We note, however, that, as noted earlier, very similar ideas have been explored for neutron transport eigenvalue calculations [40, 42, 15, 43, 51, 45, 33].

\subsection{Thermal radiative transfer equation}

We are interested in obtaining the solution of the following thermal radiative transfer (TRT) equation [26],

$$
\frac{1}{c} \frac{\partial I}{\partial t}+\mathbf{\Omega} \cdot \nabla I+\sigma I=\sigma B
$$

where $I=I(\mathbf{r}, \Omega, v, t)$ is the specific radiation angular intensity, $\sigma=\sigma(v, T)$ is the opacity, and $B=B(v, T)$ is the black-body reemission source (Eq. 5). Both $\sigma$ and $B$ are generally highly varying functions of frequency, $v$ and material temperature, $T$. The energy evolution in the participating media can be described by the following equation,

$$
\rho c_{v} \frac{\partial T}{\partial t}=\int_{0}^{\infty} d v \int_{4 \pi} d \Omega(\sigma I-\sigma B)=\sigma_{E} c E_{r}-\sigma_{P} a c T^{4},
$$

where $\sigma_{E}=\frac{\int_{0}^{\infty} \int_{4 \pi} \sigma I d \Omega d v}{\int_{0}^{\infty} \int_{4 \pi} I d \mathbf{\Omega} d v}$ and $\sigma_{P}=\frac{\int_{0}^{\infty} \int_{4 \pi} \sigma B d \Omega d v}{\int_{0}^{\infty} \int_{4 \pi} B d \Omega d v}$ are the radiation-energy-density-weighed and Planckweighed opacities, respectively, $\rho$ and $c_{v}$ are the density and specific heat of material, and $E_{r}=\frac{1}{c} \int d v \int d \Omega I$ is the radiation energy density. Convergence of the fixed-point iteration can be very slow when absorption, $\sigma I$, and reemission, $\sigma B$, are tightly coupled. To accelerate convergence of the absorption-reemission physics, a moment-based acceleration can be used [16, 27, 28, 30,31]. Integrating over all frequency, and taking the first two angular moments together with a material energy equation, yields the following gray LO system:

$$
\begin{aligned}
\frac{\partial E_{r}}{\partial t}+\nabla \cdot \mathbf{F}+\sigma_{E} c E_{r} & =\sigma_{P} a c T^{4} \\
\frac{1}{c} \frac{\partial \mathbf{F}}{\partial t}+\frac{c}{3} \nabla c E_{r}+\sigma_{R} \mathbf{F} & =\gamma c E_{r} \\
\rho c_{v} \frac{\partial T}{\partial t}+\sigma_{P} a c T^{4}-\sigma_{E} c E_{r} & =0
\end{aligned}
$$

where $\mathbf{F}=\int \Omega I d \Omega d v$ is the (gray) radiative flux. The equations are closed at the second moment (Eddington tensor), but with two further approximations in Eq. 10. First, the Eddington tensor is replaced by $\mathcal{I} / 3$, which is analogous to Fick's diffusion law. Second, we use the Rosseland-weighted opacity, $\sigma_{R}$, in order to avoid nonphysical opacity values.

The inconsistency and discretization/truncation error mismatch between $\mathrm{HO}$ and $\mathrm{LO}$ systems are accounted for by the consistency term $\gamma$ in Eq. 10 [16]. The HO solution is used to evaluate the consistency term $\gamma$ by substituting the $\mathrm{HO}$ solution (obtained by solving Eq. 7 with current LO values for $\sigma$ and $B$ ) into the discrete form of Eq. 10. Thus, the HO solver is nonlinearly eliminated via the consistency term. With this 
definition of $\gamma$, the LO system is guaranteed to have the same local energy conservation property as the HO system. The coupled LO system can be efficiently solved via a preconditioned Newton-Krylov method $[21,22,27]$.

\subsection{Numerical Examples}

\subsubsection{D gray Marshak wave problem}

The first example problem is a 1D gray Marshak wave problem [27]. The problem consists of a homogeneous material with a length of $2.0 \mathrm{~cm}$. We use a uniform mesh size of $0.02 \mathrm{~cm}$. An isotropic incoming radiation intensity of

$$
I(\boldsymbol{\Omega})=\frac{a c T^{4}}{4 \pi}, \mathbf{n} \cdot \mathbf{\Omega}<0,
$$

is applied on the left boundary $(\mathrm{x}=0.0 \mathrm{~cm})$. The boundary condition is linearly increased up to $150 \mathrm{eV}$ during $0<t<10^{-9} \mathrm{~s}$ and kept constant at $150 \mathrm{eV}$ for the rest of the simulation. We used a vacuum boundary condition,

$$
I(\Omega)=0, \mathbf{n} \cdot \Omega<0,
$$

at the right boundary $(x=2.0 \mathrm{~cm})$. Initially the system is in equilibrium at $0.025 \mathrm{eV}$. The opacity is temperaturedependent with the following formula,

$$
\sigma(T)=\frac{10^{6} \rho}{T^{3}}
$$

where the material density is set to $1.0 \mathrm{~g} / \mathrm{cm}^{3}$. The specific heat capacity is set to $1.3784 \times 10^{11} \mathrm{erg} / \mathrm{eV}-\mathrm{g}$.

Figs. $1 \mathrm{a}$ and $1 \mathrm{~b}$ compare the error in the radiation temperature, $T_{r}=\left(E_{r} / a\right)^{1 / 4}$, and material temperature at $t=5 \times 10^{-8} \mathrm{~s}$. We use a solution obtained by Picard/source iterations (SI) with a constant $\Delta t=5 \times 10^{-13} \mathrm{~s}$ as the base solution to compute the error. For this time-step range, predictor-corrector (PC) yields second-order convergence behavior in time, while the convergence of the linearization-based Implicit Monte Carlo (IMC) method of Fleck and Cummings (FC) [52] is first-order. Quantitatively, the PC algorithm can reduce the error by $1.5 \sim 4$ orders of magnitude compared to the FC case for similar $\Delta t$. The error in the PC algorithm is slightly larger than that of SI. SI directly solves the Crank-Nicolson (CN) time-discrete version of the couple system Eqs. 7 and 8 with a Picard iteration. Convergence of SI is measured with the relative tolerance, $t o l=\left\|I^{k}-I^{k-1}\right\| /\left\|I^{k}\right\|$. An average of 10 transport sweeps is required to reduce the error to $t o l=10^{-3}$ with a time step size of $\Delta t=10^{-10} \mathrm{~s}$.

Note that not only does the PC algorithm have a smaller error for a given time step size compared to the FC method, but the PC algorithm is the most efficient per time step of the three methods (i.e., PC, FC and SI) because PC only requires one "transport sweep", while FC and SI require one "transport solve" (i.e., converging the nonlinear couplings in Eqs. 7, 8 in SI, or solving for the integral coupling of the effective scattering source in FC) per time step.

\subsubsection{Monte Carlo HO solver extension}

Next, we present the use of Monte Carlo simulation methods, which may be advantageous in certain situations. The combination of energy-conserving tallies and the use of asymptotic approximation in op- 


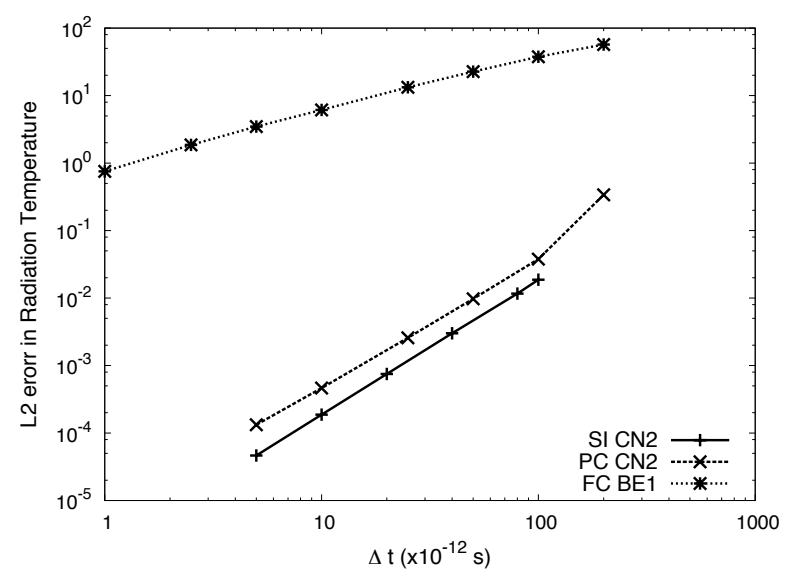

(a) Radiation Temperature

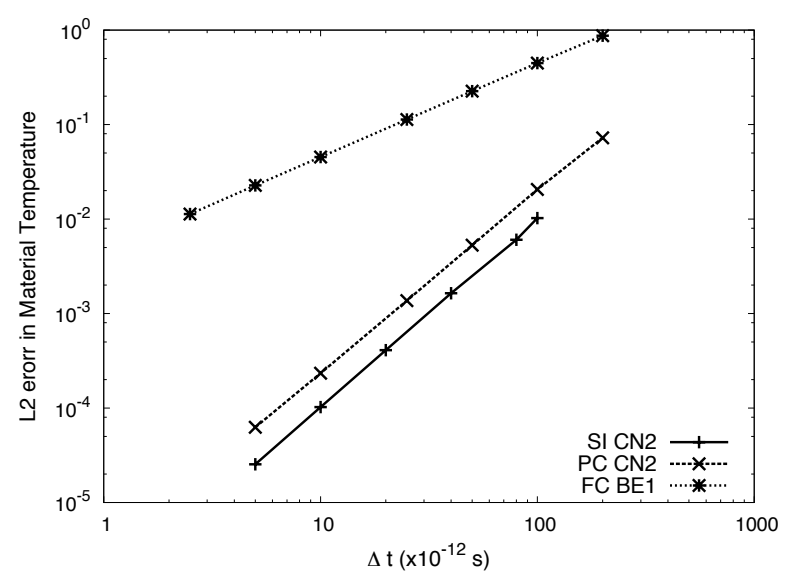

(b) Material Temperature

Figure 1: Time step convergence results for the 1D, grey Marshak wave problem.

tically thick region alleviate the difficulties of local energy conservation and mitigation of statistical noise [31]. To demonstrate efficiency and accuracy of the method, we solve the 2D crooked pipe problem [53] and compare against IMC. Although the original problem is an axisymmetric cylindrical RZ problem, we use a Cartesian XY geometry. The problem consists of an optically thin region and optically thick regions, with temperature-independent opacities of $\sigma=0.2$ and $2000 \mathrm{~cm}^{-1}$, respectively. The problem domains spans $0<x<2 \mathrm{~cm}$ and $0<y<7 \mathrm{~cm}$. The system is initially in equilibrium at $50 \mathrm{eV}$. A $500 \mathrm{eV}$ isotropic surface source is applied at $x<0.5 \mathrm{~cm}, y=0 \mathrm{~cm}$. We used a uniform mesh size of $0.025 \mathrm{~cm}$ in both $\mathrm{x}$ and $\mathrm{y}$ directions. The initial time-step size was set to $10^{-11} \mathrm{~s}$ and maximum time-step size was set to $10^{-9} \mathrm{~s}$. On average, $10^{6}$ Monte Carlo particles were used to simulate each HO step.

Fig. 2 depicts the radiation temperature at $5 \times 10^{-7} \mathrm{~s}$. The left solution was obtained with the IMC method, while the right solution was obtained using the HOLO Monte Carlo method with the $O(\epsilon)$ asymptotic approximation [31]. To reach a simulation time of $5 \times 10^{-7} \mathrm{~s}$, the IMC method required about 138 CPU-hours, while the HOLO Monte Carlo with $O(\epsilon)$ asymptotic approximation required about 3 CPUhours. Fig. 3a shows the CPU time vs. simulation time for both IMC and HOLO Monte Carlo. At the beginning of the simulation, IMC is faster than HOLO Monte Carlo. This is due to the whole system being relatively cold and thus exhibiting correspondingly little effective scattering. Thus, there are very few effective-scattering events. At this point, the CPU time corresponding to the LO solve is still significant. However, once the system has heated up and there are a large number of effective-scattering events, IMC requires significant CPU effort each time step. On the other hand, Fig. $3 \mathrm{~b}$ shows that HOLO Monte Carlo requires a relatively constant CPU effort per time step (grind time) regardless of the problem evolution. The computational effort of our HOLO algorithm is very similar to that of hybrid IMC/DDMC [54]. For this 2D crooked pipe problem, we observed a 10\% difference in CPU time between the two approaches depending on input parameters. However, we would like to emphasize that our HOLO algorithm con- 


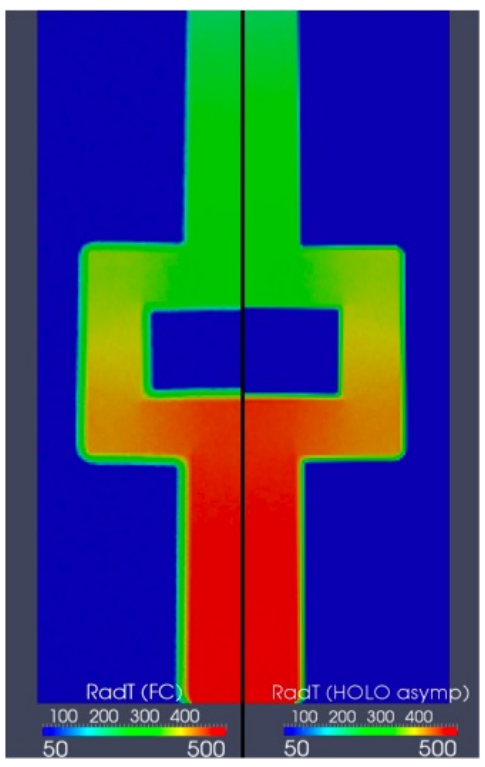

Figure 2: Comparison of radiation temperature solution at $t=50$ sh for the 2D XY crooked pipe problem. Left: the solution from IMC. Right: the solution from HOLO Monte Carlo with the $O(\epsilon)$ asymptotic approximation.

verges the absorption/re-emission physics nonlinearly in both optically thin and optically thick regions, whereas IMC (and DDMC) employs effective scattering. In addition, the availability of the LO system may be advantageous when additional physics is combined with thermal radiative transfer.

\subsubsection{Residual Monte Carlo HO solver}

One advantage of the HOLO algorithm is that we often have a good approximated solution computed by the LO system. In this case, asking a (standard) Monte Carlo simulation to resolve the end of time-step solution may be inefficient. Assuming we have a good approximated solution, $I^{+}$, the transport equation can be rewritten in the following residual form,

$$
\frac{\delta^{n+1}}{c \Delta t}+\boldsymbol{\Omega} \cdot \nabla \delta^{n+1}+\sigma \delta^{n+1}=\frac{\sigma a c\left(T^{n+1}\right)^{4}}{4 \pi}+\frac{I^{n}}{c \Delta t}-\left(\frac{I^{+}}{c \Delta t}+\mathbf{\Omega} \cdot \nabla I^{+}+\sigma I^{+}\right),
$$

where $\delta^{n+1}=I^{n+1}-I^{+}$. Since the error in the simulation is directly proportional to the magnitude of the source term, the statistical noise incurred in computing $\delta^{n+1}$ is smaller than computing $I^{+}$directly via standard Monte Carlo method. In order to analyze and compare Residual Monte Carlo algorithm, we first define Figure of Merit (FoM) which acts as a measure of efficiency of each algorithm,

$$
\text { FoM }=\frac{1}{\| \text { Error } \|_{2} \times \text { PPTS }}
$$

where PPTS refers to the average number of particles per transport sweep.

We solve a 1D gray two-material problem to demonstrate effectiveness of Residual Monte Carlo HO solver [28]. This problem represents a multi-material domain in which there exists a sharp material interface. Half way through domain there is a transition from an optically thin material to optically thick material. We consider a $1.0 \mathrm{~cm}$ domain which is initially in equilibrium at $50 \mathrm{eV}$. We apply $500 \mathrm{eV}$ isotropic 


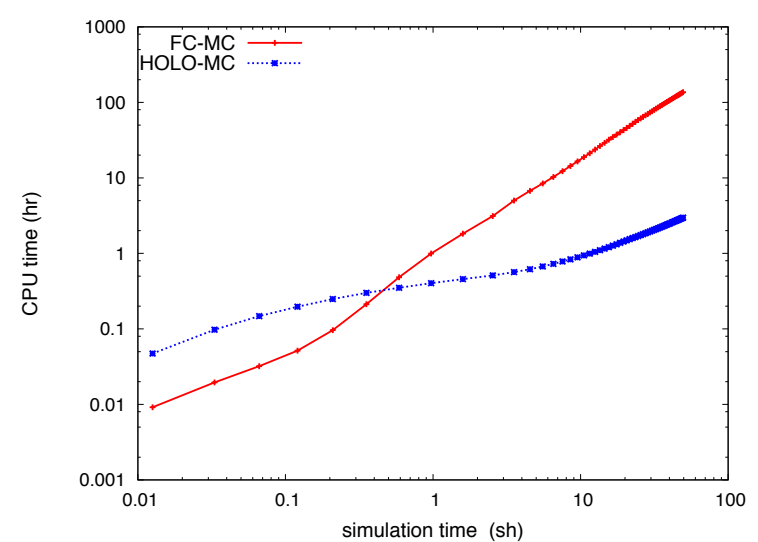

(a) CPU vs simulation time

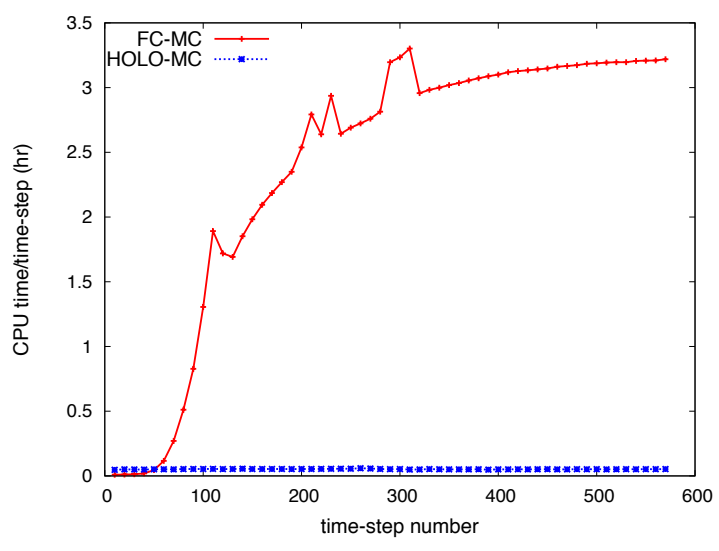

(b) CPU per every 10 time step vs time-step number

Figure 3: Comparison of CPU time vs simulation time for the 2D crooked pipe problem.

Table 1: Material properties for 1D 2material problem.

\begin{tabular}{ccc}
\hline & Material 1 & Material 2 \\
\hline \hline x-range & $<0.5 \mathrm{~cm}$ & $>0.5 \mathrm{~cm}$ \\
$\sigma\left[\mathrm{cm}^{-1}\right]$ & 0.2 & 2000 \\
$\rho\left[\mathrm{g} / \mathrm{cm}^{3}\right]$ & 0.01 & 10.0 \\
$\mathcal{c}_{v}[\mathrm{erg} / \mathrm{eV} \mathrm{g}]$ & $10^{12}$ & $10^{12}$ \\
\hline
\end{tabular}

source at the $x=0$. The material properties are summarized in Table 1 . We use 64 angular bins and 100 spatial cells. The FoMs for this problem are presented in Table 2. For each choice of Monte Carlo particles, we see that RMC provides a significant performance improvement over SMC. The FoMs for this problem can reach over 1000 when RMC is used. This can be explained because there is a strong emission source that needs to be simulated even beyond the radiation front. SMC requires particles to be distributed everywhere, while RMC can effectively place the Monte Carlo particles where solution is far from equilibrium. Finally, Fig. 4 compares spatial distribution of errors between SMC and RMC.

\section{Application 2: Gas dynamics}

In this section, we extend the HOLO concept introduced in the previous section to develop a powerful new accelerator algorithm for the BGK gas dynamics Boltzmann transport equation.

Table 2: FoM for two-material problem using 100 spatial cells.

\begin{tabular}{ccc}
\hline Method & Particles & FoM \\
\hline \hline SMC & 500 & 1 \\
RMC & 500 & 4583.2 \\
SMC & 5000 & 1.189 \\
RMC & 5000 & 3031.6 \\
SMC & 50000 & 0.340 \\
RMC & 50000 & 680.9 \\
\hline
\end{tabular}



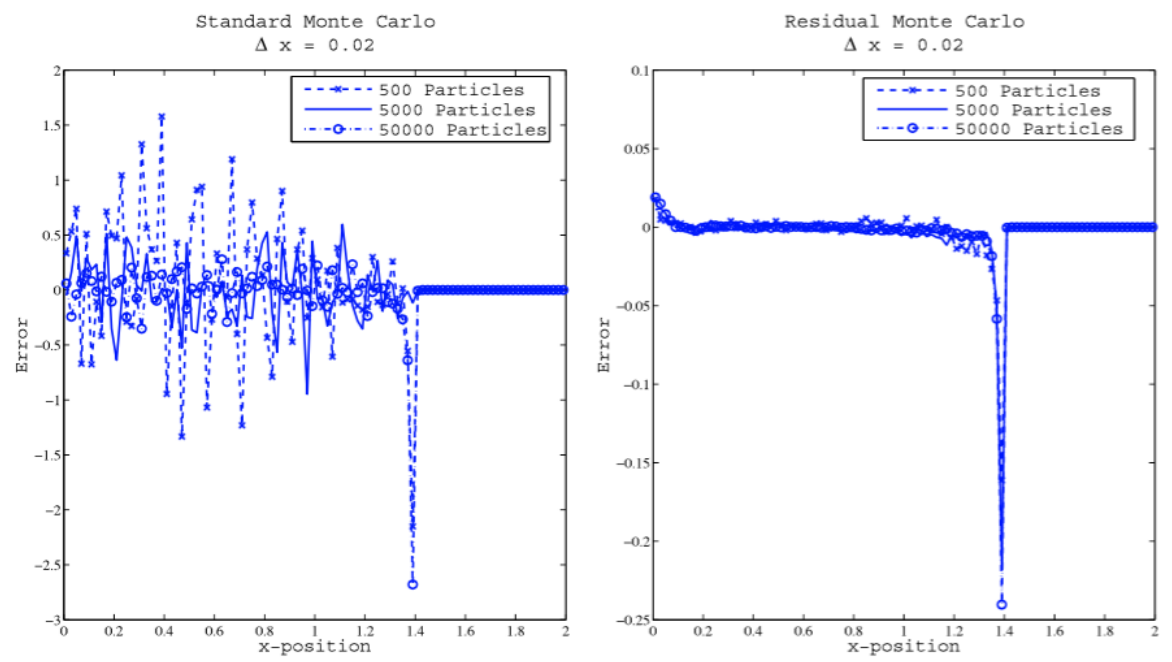

Figure 4: Comparison of SMC and RMC at $\Delta x=0.02 \mathrm{~cm}$. Note the different y-axis scales

\subsection{The gas dynamics model}

The kinetic equation of interest is the time-dependent neutral gas dynamics Boltzmann transport equation,

$$
\frac{\partial f}{\partial t}(\mathbf{r}, \mathbf{v}, t)+\mathbf{v} \cdot \nabla f(\mathbf{r}, \mathbf{v}, t)=\left(\frac{\partial f}{\partial t}\right)_{c} .
$$

Here, $f$ is the distribution function of particles in the gas, which is a function of the configuration space, $\mathbf{r}$, the particle velocity, $\mathbf{v}$, and time, $t$. The terms on the left hand side represents the time rate of change of $f$ and local change of $f$ due to the streaming of the particle. The right hand side term is the collision operator. In this study, a simple BGK collision operator [55] is used,

$$
\left(\frac{\partial f}{\partial t}\right)_{c}=\frac{1}{\tau(\mathbf{r}, t)}\left[f_{M}(\mathbf{r}, \mathbf{v}, t)-f(\mathbf{r}, \mathbf{v}, t)\right] .
$$

Here, $\tau$ is the collision time scale,

$$
\tau=\frac{\tilde{\tau}}{n \sqrt{T}}
$$

and $f_{M}$ is a local Maxwellian distribution,

$$
f_{M}(\mathbf{r}, \mathbf{v}, t)=\frac{n}{(2 \pi T / m)^{d / 2}} \exp \left\{-\frac{m}{2 T} \sum_{\alpha}\left(v_{\alpha}-u_{\alpha}\right)^{2}\right\},
$$

with $\widetilde{\tau}$ a reference collisional time scale, $d$ the translational degrees of freedom of the gas molecule, and $\alpha$ the index for the direction of the particle $(\alpha=x, y$, or $z)$. The Maxwellian distribution is a function of the local number density, $n$, the average fluid velocity, $\mathbf{u}$, and the temperature, $T$, which are velocity moments of the distribution function defined as,

$$
n=n(\mathbf{r}, t)=\langle 1, f\rangle_{\mathbf{v}}
$$




$$
\begin{gathered}
\mathbf{u}=\mathbf{u}(\mathbf{r}, t)=\frac{\langle\mathbf{v}, f\rangle_{\mathbf{v}}}{n}, \\
T=T(\mathbf{r}, t)=\frac{1}{d} m \frac{\left\langle|\mathbf{w}|^{2}, f\right\rangle_{\mathbf{v}}}{n} .
\end{gathered}
$$

Here, $m$ is the mass of the particle, $\mathbf{w}$ is the thermal fluctuating random component of the velocity,

$$
\mathbf{w}=\mathbf{v}-\mathbf{u},
$$

and $\langle(\cdot), f\rangle_{\mathbf{v}}$ is a shorthand notation for the velocity moment, i.e.,

$$
\langle(\cdot), f\rangle_{\mathbf{v}}=\iiint(\cdot) f d \mathbf{v}
$$

The BGK collision operator will effectively relax the distribution to a Maxwellian in a few collision times, $\tau$. In this study, we consider the one dimensional in space $(x)$ and one dimensional in velocity space $(v)$ $-1 \mathrm{D} 1 \mathrm{~V}-$ model of Eq. 17,

$$
B B G K=\frac{\partial f}{\partial t}(x, v, t)+v \frac{\partial f}{\partial x}(x, v, t)=\frac{1}{\tau}\left[f_{M}(x, v, t)-f(x, v, t)\right] .
$$

The equation supports time-scales ranging from the stiff collision time to the slower acoustic wave time scale. To implicitly solve problems where the collision time scale and acoustic time scales are on the same scale, classic fixed point iteration schemes [56] may be used. However, in transitional flow problems where regions of both rarefied and dense gas coexist in the same domain, one would be interested in stepping over the collision time scales of the dense region and follow the dynamical acoustic time scale of the problem. For this case, $\Delta t / \tau \gg 1$ and the fixed-point schemes will require a large number of iterations, to the point that the method becomes intractable. Newton's method is a possible alternative to control the iteration count, but is challenged by the BBGK equation's integro-differential nature. The challenge resides in the efficient and accurate construction and inversion of the required Jacobian matrix, which is critical for the scalability and convergence of the method. In the next section, we discuss a HOLO algorithm solution for the current problem, which uses the LO moment equation to nonlinearly accelerate the convergence of the classic fixed-point iteration scheme.

\subsection{HOLO algorithm}

To implicitly evaluate the BGK collision operator, one needs the density, $n$, the drift velocity, $u$, and temperature, $T$. To accelerate the convergence of the standard fixed point acceleration scheme, we introduce a discretely-consistent lower-order (LO) moment equation to provide an improved implicit estimate for these collisional coefficients. To derive the LO self-consistent fluid moment equations, we take the $1, v$, and $\frac{v^{2}}{2}$ moment of the 1D1V Boltzmann transport equation in Eq. 19,

$$
\left\langle v^{0}, B B G K\right\rangle_{v}=\frac{\partial n}{\partial t}+\frac{\partial}{\partial x} n u=0
$$




$$
\begin{gathered}
\left\langle v^{1}, B B G K\right\rangle_{v}=\frac{\partial}{\partial t} n u+\frac{\partial}{\partial x}\left[n u^{2}+\frac{P}{m}\right]=0 \\
\left\langle\frac{v^{2}}{2}, B B G K\right\rangle_{v}=\frac{\partial}{\partial t} n E+\frac{\partial}{\partial x}\left[u\left(n E+\frac{P}{m}\right)+\frac{q}{m}\right]=0 .
\end{gathered}
$$

Here, as before, $n$ is the density, $u$ is the bulk velocity, $n E$ the specific total energy density,

$$
n E=n E(x, t)=\left\langle\frac{v^{2}}{2}, f\right\rangle_{v}
$$

$P$ the scalar pressure,

$$
P=m\left\langle w^{2}, f\right\rangle_{v}=m\left\langle(v-u)^{2}, f\right\rangle_{v}=n T
$$

and $q$ the heat flux,

$$
q=\frac{m}{2}\left\langle w^{3}, f\right\rangle_{v} .
$$

Equations 20, 21, and 22 are the continuity, momentum, and energy equation, respectively. In standard gas dynamics, the heat flux is closed with a Fourier model for heat conduction, $q=-\kappa \frac{\partial T}{\partial x}$, and one is required to provide a functional form for $\kappa$. We will not do that here. Rather, we will close this heat-flux using the most recent iteration level of the distribution function.

Our HOLO algorithm is as follows. The HO solution $f$ is advanced using the current LO solution state, which defines the collision operator as:

$$
\frac{\partial f}{\partial t}+v \frac{\partial f}{\partial x}=\frac{f_{M}^{L O}-f}{\tau^{L O}}
$$

where

$$
f_{M}^{L O}=\frac{n^{L O}}{\sqrt{2 \pi T^{L O} / m}} e^{-\frac{m\left(v-u^{L O}\right)^{2}}{2 T^{L O}}}
$$

and

$$
\tau^{L O}=\frac{\tilde{\tau}}{n^{L O} \sqrt{T^{L O}}} .
$$

Once the new $f$ is found, it is used to close the LO moment system (in this case through the heat flux, $q^{\mathrm{HO}}$ ), so that a new LO solution state can be found. As in the radiation transport application, consistency terms enforce discrete consistency between $\mathrm{HO}$ and LO formulations [17]. The consistency terms are introduced in the LO moment equations as follows:

$$
\begin{gathered}
\frac{\partial n^{L O}}{\partial t}+\frac{\partial}{\partial x} n u^{L O}-\frac{\partial \gamma_{n}^{H O}}{\partial x}-\mathcal{I}_{n}^{H O}=0, \\
\frac{\partial}{\partial t} n u^{L O}+\frac{\partial}{\partial x}\left[\left(n u^{L O}\right)^{2}+P^{L O}\right]-\frac{\partial \gamma_{n u}^{H O}}{\partial x}-\mathcal{I}_{n u}^{H O}=0, \\
\frac{\partial}{\partial t} n E^{L O}+\frac{\partial}{\partial x}\left[u\left(n E^{L O}+P^{L O}\right)+q^{H O}\right]-\frac{\partial \gamma_{n E}^{H O}}{\partial x}-\mathcal{I}_{n E}^{H O}=0 .
\end{gathered}
$$

Here, the $\mathrm{HO}$ superscript denotes that the quantity comes from the evaluation of the distribution function. The $\gamma^{H O}$ and $\mathcal{I}^{H O}$ are the consistency terms, which ensure that, upon convergence, $n^{L O}=n^{H O}, n u^{L O}=$ 
$n u^{H O}$, and $n E^{L O}=n E^{H O}$. The flux consistency terms $\gamma^{H O}$ are computed as the difference between the HO moments of the streaming flux and the Euler component of the conservative flux (evaluated in terms of $\mathrm{HO}$ quantities) [17]. The collisional consistency terms $\mathcal{I}^{H O}$, on the other hand, are computed as the HO moment of the BGK collision operator, and take into account the fact that collisional conservation properties are not strictly ensured for a truncated velocity-space domain.

In practice, our nonlinear solver iterates on the LO problem, with the HO problem Picard-linearized, and enslaved to provide $q^{\mathrm{HO}}$ and the consistency terms, $\gamma^{\mathrm{HO}}$ and $\mathcal{I}^{\mathrm{HO}}$.

\subsection{Numerical results}

We discuss the performance of the new HOLO algorithm applied to several test cases: 1) a SOD shock tube problem [57] and 2) a strong shock problem with a widely varying characteristic collision time-scale. Before beginning the discussion on the test problems, a word is in order about our discretization. In time, we adopt an asymptotic preserving generalized theta scheme while in space, we adopt a finite volume discretization in both the HO and LO system. A monotonicity preserving ULTRA-SHARP+WENO discretization is considered for the advection operator for the $\mathrm{HO}$ system. The full details of discretization can be found in Ref. [17].

\subsubsection{SOD shock tube}

First, we will compare the performance of the accelerated algorithm with the standard Picard iteration algorithm for a well known SOD shock tube problem [57] for different values of collision time-scale, $\tau$. We test the algorithm for a constant $\tau$ everywhere in the domain. The system length is $L_{x}=1$ in a dimensionless unit and the initial diaphragm is situated at $x=0.5 L_{x}$. Consider the following initial condition for the SOD shock tube,

- $n_{L}=1.0, n_{R}=0.125$,

- $T_{L}=1.0, T_{R}=0.8$,

- $u_{L}=0.0, u_{R}=0.0$,

where the subscripts $L$ and $R$ denote the values of the flow variable immediately to the left and right of the diaphragm. The kinetic initial condition is assumed to be a Maxwellian, based on the moment values to the left and right side of the diaphragm,

$$
f_{0}=f(x, v, t=0)=\frac{n_{0}}{\sqrt{2 \pi T_{0} / m}} \exp \left[-\frac{m}{2 T_{0}}\left(v-u_{0}\right)^{2}\right] .
$$

For details of numerical parameters and convergence criteria, readers are referred to Ref. [17].

Five cases of the SOD shock tube were run with a fixed time-step size, $\Delta t=5 \times 10^{-4}$, and fixed collision time-scales $\tau=5 \times 10^{-6}, 5 \times 10^{-5}, 5 \times 10^{-4}, 5 \times 10^{-3}$, and $5 \times 10^{-2}$ (i.e., Eq. 18 is not employed). The motives to consider these five cases are two fold: 1) to demonstrate the performance of the standard linearized

Picard iteration scheme as a function of $\frac{\Delta t}{\tau}$ and how it compares to the moment accelerated scheme, and 
Table 3: Performance comparison of HOLO moment acceleration scheme versus the standard scheme

\begin{tabular}{cccccc}
\hline$\Delta t / \tau$ & $10^{-2}$ & $10^{-1}$ & $10^{0}$ & $10^{1}$ & $10^{2}$ \\
\hline \hline Iteration $_{\text {HOLO }}$ & 4 & 6 & 8 & 9 & 9 \\
Iteration $_{\text {standard }}$ & 5 & 13 & 68 & 461 & 2853 \\
\hline
\end{tabular}

2) to demonstrate how the moment accelerated scheme can be used to follow the acoustic time-scale rather than the collision time-scale.

In order to highlight the time-scale that the moment acceleration scheme allows one to follow, we compare the $\Delta t$ size with respect to the acoustic wave CFL for the $\tau=5 \times 10^{-6}$ case. The maximum speed of sound during the entire duration of the simulation is $c \approx 2.3$. The acoustic wave CFL is defined as:

$$
\Delta t_{A-C F L}=\frac{\Delta x}{c}
$$

For the mesh considered in this problem [17] this equates to a $\Delta t_{A-C F L}=8.7 \times 10^{-4}$. This equates to a chosen time-step size of, $\Delta t=0.3 \Delta t_{A-C F L}$. If one used a standard Picard iteration, one would have required to run at the collisional time scale, $\Delta t=\tau=5 \times 10^{-6}=2.8 \times 10^{-3} \Delta t_{A-C F L}$, which is almost two orders of magnitude smaller than what we have used with the HOLO algorithm.

The performance of the algorithm is shown in Table 3 for the five cases. The first and second row presents the number of $\mathrm{HO}$ solves required for the moment acceleration algorithm and the standard algorithm, respectively. For a small $\frac{\Delta t}{\tau}, \Delta t$ respects the collision time scale and the convergence rate of the two methods is not too different. However, as the ratio $\frac{\Delta t}{\tau}$ increases, the standard scheme requires many more iterations in order to converge the BBGK equation, while for the HOLO scheme the increase is much slower and plateaus. For the $\Delta t / \tau=10^{2}$ case, the moment acceleration algorithm shows a reduction in the number of transport sweeps that is more than two orders of magnitude.

\subsubsection{Strong shock tube with variable $\tau$}

Finally, we perform a simulation with a strong shocktube problem with a solution-dependent collision time-scale. In this example, we show the true multi-scale nature of the BBGK equation for problems with a transition layer and a realistically varying collision timescale, where a wide separation in Knudsen number (defined as the ratio between the mean free path $\lambda_{m f p}$ and the gradient length scale $L_{T}$ of the evolving solution) [58] exists throughout the domain. In this study, we define the Knudsen number as,

$$
K_{n}=\frac{\lambda_{m f p}}{L_{T}}
$$

where,

$$
L_{T}=\left\|\left(\frac{1}{T} \frac{\partial T}{\partial x}\right)^{-1}\right\| .
$$

For a variable collision time-scale, we also have a varying mean-free-path throughout the domain. For a strong shock, the difference in mean-free-path across the domain may be orders of magnitude. This allows for a large separation in length scales within the shock layer. For hydrodynamic models to give an accurate 
Table 4: Performance comparison of moment acceleration scheme versus the standard scheme for a strong shock problem

\begin{tabular}{ccccc}
\hline$\Delta t / \tau_{\min }$ & $5 \times 10^{0}$ & $1.25 \times 10^{1}$ & $5 \times 10^{1}$ & $5 \times 10^{2}$ \\
\hline \hline Iteration $_{\text {HOLO }}$ & 4 & 4 & 4 & 10 \\
Iteration $_{\text {standard }}$ & 51 & 108 & 402 & $>1000$ \\
\hline
\end{tabular}
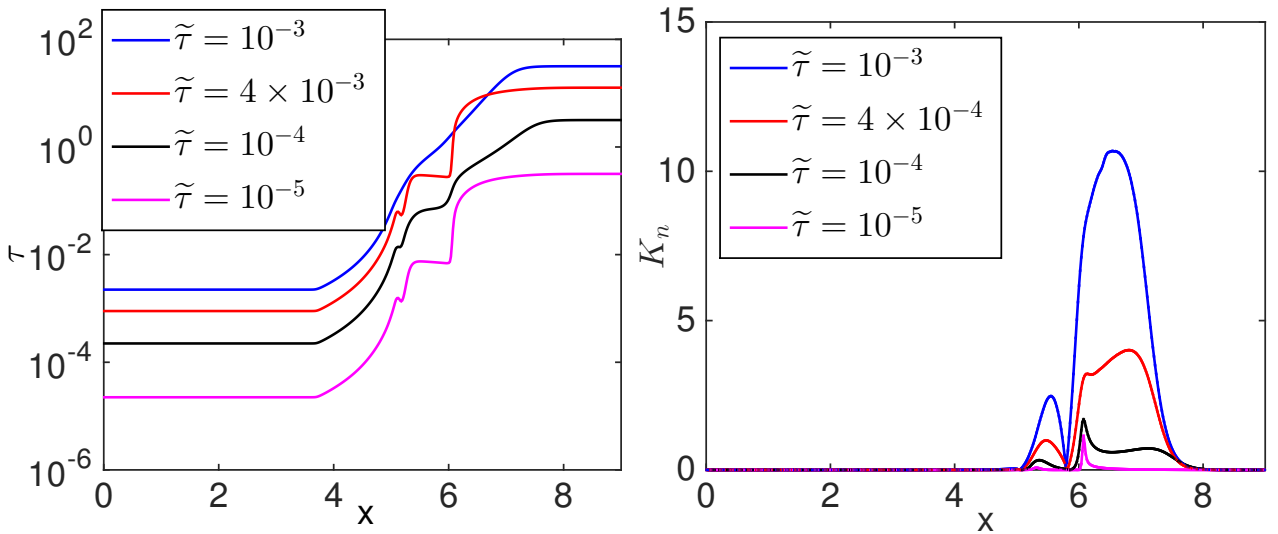

Figure 5: Comparison of $\tau$ and $K n$ for different $\widetilde{\tau}$ for strong shock tube.

solution, $K n=\lambda_{m f p} / L_{T} \ll 1$. If $\lambda_{m f p}$ is larger than $L_{T}$, a large physical dissipation will be introduced, which cannot be captured accurately with a hydrodynamic model. From an algorithmic point of view, the standard iteration scheme for the $\mathrm{HO}$ solver will incur many $\mathrm{HO}$ evaluations when $\Delta t / \tau_{\min } \gg 1$, with $\tau_{\min }$ the smallest $\tau$ in the shock layer. This is exactly the regime where our new algorithm will produce an accurate solution, efficiently.

For the strong shock problem, the system domain of $L_{x}=[0,9]$ is chosen with a simulation time of $t=[0,1]$. The following initial condition is used,

- $n_{L}=1.0, n_{R}=10^{-3}$,

- $P_{L}=0.1, P_{R}=10^{-6}$,

- $u_{L}=0, u_{R}=0$.

We test the shock tube problem with $\tau$ defined by equation (18), and $\widetilde{\tau}=10^{-3}, 10^{-4}, 4 \times 10^{-4}$, and $10^{-5}$. In Table 4, the performance of the HOLO moment acceleration scheme and the standard Picard iteration is compared. As can be seen, the moment acceleration method requires significantly fewer evaluations of the HO system. In Fig. 5, we show the $\tau$ and the $K n$ in log-scale for the four $\tilde{\tau}$ cases late in time, when the problem has evolved. For all cases of $\tilde{\tau}$, we see a separation of about four orders of magnitude in $\tau$. For this set of simulations, convergence of the standard iterative solver will suffer for $10^{-5} \leq \widetilde{\tau} \leq 10^{-4}$, since for these cases $\Delta t / \tau_{\min } \gg 1$. Hydro models will break down for $\widetilde{\tau} \geq 10^{-4}$ since $K_{n} \approx \mathcal{O}(1)\left(K_{n} \ll 1\right.$ is required for the hydrodynamic models to be accurate). For this reason, hydro models are generally not suitable for strong shocks, and a kinetic model is required. The new HOLO moment acceleration algorithm is specifically designed for these transition-flow problems. 


\section{Application 3: Plasma kinetic HOLO algorithms}

\subsection{Collisionless Particle-in-cell HOLO algorithms}

Kinetic simulation of plasmas is of importance in hot and/or rarified conditions such as those in magnetically confined fusion plasmas and the Earth's magnetophere. Collisionless plasmas are described by the Vlasov-Maxwell set of equations, or a subset thereof. The Vlasov-Maxwell equation set is high dimensional (6D), and multiscale, supporting disparate time and length scales.

Particle-in-cell (PIC) algorithms were developed starting in the 50s to attack the curse of dimensionality of the Vlasov-Maxwell equation by considering a collection of characteristics ("particles") to solve the Vlasov equation $[59,60]$, and have proved to be very successful for first-principles simulations of plasma dynamics. The classical PIC method employs an explicit approach (e.g. leap-frog) to advance the VlasovMaxwell/Poisson system using particles coupled to a grid. Explicit PIC is subject to both temporal stability constraints (either light-wave CFL condition or resolving plasma-wave frequency) and spatial stability (so-called finite-grid instability, which requires resolution of the smallest length scale, the electron Debye length) constraints. Furthermore, numerical heating due to the lack of exact discrete energy conservation $[59,60]$ compromises the accuracy of explicit PIC simulations over long time scales, particularly for realistic ion-to-electron mass ratios, severely limiting its use for system-scale kinetic simulations, even with modern super-computers.

Motivated by the need to overcome these limitations, there has been a long tradition of development of multiscale, implicit moment-based acceleration methods for PIC [5, 6, 7, 8, 11, 12, 61, 18, 19, 20]. Implicit PIC algorithms can potentially eliminate both spatial and temporal stability constraints. This motivated much exploration of these algorithms in the 1970's and 1980's $[62,5,6,7,11,63,64,65,66,8,67,68,69,70,71,72,12$, 73]. These studies explored the viability of an implicit PIC formulation and its accuracy properties, and led to the exploration of moment-accelerated algorithms such as the implicit-moment method [5, 6, 7, 8, 11, 12]. However, the unavailability of efficient nonlinear solvers for a very large system of particle-field equations demanded approximations that defeated the goal of accurate long-term simulations [74].

More recently, there has been much progress in the development of fully implicit, moment-accelerated PIC algorithms $[61,75,13,19,20]$ that are both accurate (featuring exact local charge and global energy conservation theorems) and efficient (they scale optimally with mesh refinement, and do not require resolution of the Debye length for stability). In what follows, we introduce the problem, summarize the algorithmic implementation details, and exemplify the performance of the method on representative examples. Here, we consider the so-called Darwin model, which projects out the light wave, and is suitable for the simulation of long-frequency phenomena. It should be noted, however, that the approach proposed here can be readily extended to full classical and relativistic Vlasov-Maxwell models [76]. 


\subsubsection{The Vlasov-Darwin model}

The full set of Vlasov-Darwin equations $[62,77,78,79,80,19]$ in the potential form reads:

$$
\begin{aligned}
\frac{\partial f_{\alpha}}{\partial t}+\mathbf{v} \cdot \nabla f_{\alpha}+\frac{q_{\alpha}}{m_{\alpha}}(\mathbf{E}+\mathbf{v} \times \mathbf{B}) \cdot \nabla_{v} f_{\alpha} & =0 \\
\frac{1}{\mu_{0}} \nabla^{2} \mathbf{A}+\mathbf{j}-\epsilon_{0} \nabla \frac{\partial \phi}{\partial t} & =0 \\
\epsilon_{0} \nabla^{2} \phi+\rho & =0
\end{aligned}
$$

where $f_{\alpha}(\mathbf{r}, \mathbf{v})$ is the particle distribution function of species $\alpha$ in phase space, $q_{\alpha}$ and $m_{\alpha}$ are the species charge and mass respectively, $\epsilon_{0}$ and $\mu_{0}$ are the vacuum permittivity and permeability respectively, $\phi$ and $\mathbf{A}$ are the scalar and vector potentials, respectively. For Vlasov-Darwin, only the Coulomb gauge $\nabla \cdot \mathbf{A}=0$ is physically acceptable. The electric and magnetic fields are defined uniquely from $\phi, \mathbf{A}$ as:

$$
\mathbf{E}=-\nabla \phi-\frac{\partial \mathbf{A}}{\partial t} ; \mathbf{B}=\nabla \times \mathbf{A} .
$$

Instead of the the Poisson equation, Eq. 32, we consider the equivalent form based on charge conservation,

$$
\epsilon_{0} \partial_{t} \nabla^{2} \phi-\nabla \cdot \mathbf{j}=0
$$

which is advantageous in the discrete form $[19,20]$. The set of Vlasov-Darwin (VD) equations is driven by the plasma current density

$$
\mathbf{j}=\sum_{\alpha} q_{\alpha} \int f_{\alpha} \mathbf{v} d \mathbf{v}
$$

The PIC algorithm considers a representation of the particle distribution in terms of points ("particles") in phase space with constant weight $w_{p}$,

$$
f_{\alpha}(\mathbf{r}, \mathbf{v}) \approx \sum_{p \in \alpha} w_{p} \delta\left[x-\mathbf{x}_{p}(t)\right] \delta\left[\mathbf{v}-\mathbf{v}_{p}(t)\right]
$$

In practice, the $\delta$-function in configuration space is replaced by finite-support interpolation functions, typically B-splines. Particle's positions and velocities are evolved according to the method of characteristics:

$$
\begin{aligned}
\frac{d \mathbf{x}_{p}}{d t} & =\mathbf{v}_{p}, \\
\frac{d \mathbf{v}_{p}}{d t} & =\mathbf{a}_{p}=\frac{q_{p}}{m_{p}}\left(\mathbf{E}_{p}+\mathbf{v}_{p} \times \mathbf{B}_{p}\right),
\end{aligned}
$$

where the subscript $p$ denotes a particle (or Lagrangian) quantity.

\subsubsection{The HOLO PIC algorithm}

The HOLO PIC algorithm considers a fully implicit formulation for the LO Darwin field equations,

$$
\begin{aligned}
\frac{1}{\mu_{0}} \nabla^{2} \mathbf{A}+\mathbf{j}-\epsilon_{0} \nabla \frac{\partial \phi}{\partial t} & =0 \\
\epsilon_{0} \nabla^{2} \frac{\partial \phi}{\partial t}-\nabla \cdot \mathbf{j} & =0
\end{aligned}
$$


coupled with the HO system, which is comprised by the set of particle evolution equations, Eqs. 36, 37, evolved with a multirate scheme that allows for time subcycling and proper orbit resolution without spoiling exact charge conservation $[61,81,19,20]$. Exact energy conservation can be achieved discretely when an exactly time-centered (Crank-Nicolson, $\mathrm{CN}$ ) temporal discretization scheme is employed for both particles (per subcycle) and fields $[61,81,19,20]$. All those conservation properties can be naturally generalized to curvilinear geometries [82, 83].

The coupling is done via nonlinear elimination, with only the fields A, $\phi$ explicitly considered by the nonlinear solver. For this application, we employ residual-based nonlinear iterative techniques (e.g., Jacobian-free Newton-Krylov, Anderson Acceleration), which require the evaluation of the nonlinear residual to proceed in the iteration. A typical residual evaluation step consists of four main elements:

1. Input $(\phi, \mathbf{A})$.

2. Integrate particle orbits adaptively, Eqs. 36, 37.

3. Compute moments from particles (current density, Eq. 34).

4. Compute the residual of Darwin equations, Eqs. 38, 39.

Convergence of this nonlinear system is effectively accelerated by a LO moment system, either in a linear form as a preconditioner [18, 19, 20], or as a additional nonlinear set of equations [13], in which case one has to ensure discrete consistency, as indicated in previous sections.

\subsubsection{Numerical results}

Ion acoustic wave (IAW). The IAW problem is a challenging multiscale test case because it evolves on slow (ionic) time scales, but electrons play an important role. This test case only requires the electrostatic field response.

We consider first a standing wave excited by an ion density perturbation, which oscillates owing to the compression and expansion caused by ion thermal motion, as well as to the incomplete Debye shielding of electron thermal motion. We setup the problem so that Landau damping is insignificant [61]. The goal here is to keep the wave propagating and to examine the long-term accuracy of the implicit algorithm with different particle movers. In what follows, $\Delta t$ is the field time step, and $\Delta \tau$ is the particle time step. We consider four energy-conserving implicit movers: a single-step CN mover with $\Delta t=\Delta \tau=0.25$ and $\Delta t=\Delta \tau=1$, a sub-stepped CN mover with $\Delta t=4, \Delta \tau=1$ (i.e., 4 particle sub-steps per field time step), and an adaptive charge-conserving (ACC) $\mathrm{CN}$ mover. The explicit time step is 0.1 . The IAW period is 100 time units.

Simulation results are depicted in Fig. 6. Fig. 6a depicts the evolution of the ion kinetic energy, and allows us to make several important observations. Firstly, the single-step CN with $\Delta t=1$ fails to capture the long-term IAW behavior. This is because, for this particle time step, the average distance that an electron travels is larger than a cell size, and Debye shielding is not captured accurately [84]. Decreasing the time step improves the simulation results $(\mathrm{CN}$ with $\Delta t=0.25)$, as expected, albeit still with significant 

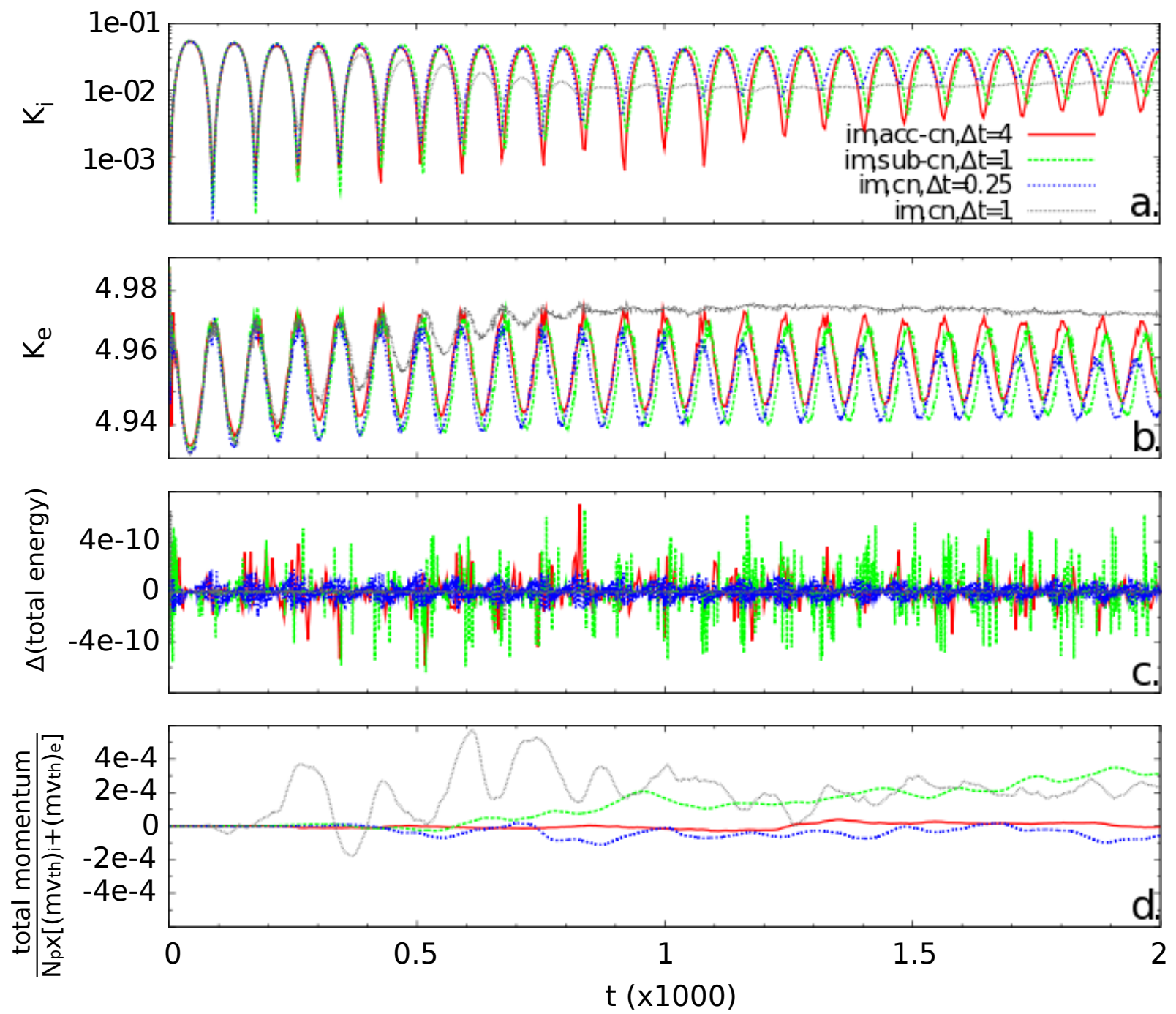

Figure 6: IAW standing-wave test. Accuracy test of the implicit algorithm using three movers: simple CN mover (cn), sub-stepped $\mathrm{CN}$ mover (sub-cn), and adaptive-charge-conserving CN mover (acc-cn). a) Time history of ion kinetic energy shows the impact of using fixed-time step movers in a long-time simulation. b) Time history of electron kinetic energy shows the importance of using small (sub-)time steps to capture the electron dynamics, which exhibits relatively small variations. c) Total energy is well conserved in all implicit simulations. d) Momentum conservation is substantially improved when particle orbits are accurately calculated using the charge-conserving self-adaptive $\mathrm{CN}$ mover. 
errors. Particle sub-stepping also improves the simulation results, as is demonstrated by the sub-stepping $\mathrm{CN}$ run, but it is still not satisfactory. The implicit solve with the ACC mover is the only one that is able to accurately capture the correct dynamics (as compared to an explicit PIC solution, not shown) despite the long field time step employed. Fig. $6 \mathrm{~b}$ shows the evolution of the electron kinetic energy. The relative magnitude of the perturbation in the electron kinetic energy is much smaller than that in the ion kinetic energy, suggesting that small errors in the electron kinetics are sufficient to ruin the accuracy of the ion wave. Fig. $6 \mathrm{c}$ and $6 \mathrm{~d}$ show the time evolution of the conserved quantities of the system, namely, total energy and total momentum, respectively. Clearly, energy is conserved exactly, while momentum is not. We see significant improvement in the momentum conservation when exact charge conservation is enforced by the ACC mover. With other movers, momentum errors accumulate in time, and the system can develop nonphysical drifts, which eventually compromise the accuracy of the IAW dynamics. These results highlight the importance of proper orbit integration and exact local charge conservation for long-term accuracy.

The importance of nonlinear convergence for long-term accuracy of the HOLO algorithm is demonstrated in Fig. 7 for a propagating IAW, which nonlinearly steepens up to become a shock wave. In this figure, we compare the fully implicit HOLO algorithm (termed "Improved IMM" in the figure) with a semiimplicit version of the algorithm lacking full convergence and discrete consistency terms in the formulation (termed "Standard IMM"). The improved IMM simulation uses a $\Delta t=100 \Delta t_{\text {exp }}$, while the standard IMM uses a time step 20 times smaller, $\Delta t=5 \Delta t_{\text {exp }}$. Fig. 7 shows the resulting electric field at various times in the simulation. As can be seen, the standard IMM evolves the field accurately in the beginning, but numerical error overwhelms the solution in the long term. In contrast, the fully implicit, charge and energy conserving version is able to find the correct nonlinear state, despite the fact that it is employing an implicit time step 20 times larger.

Weibel instability. The Weibel instability exploits free energy present in temperature anisotropy to develop a finite magnetic field in an initially unmagnetized plasma. We employ this multidimensional (2D) test case, as set up in Ref. [20], to demonstrate the efficiency advantage of the HOLO PIC method vs. explicit PIC. The efficiency advantage of the implicit PIC approach vs. the explicit one can be estimated as [19, 20]:

$$
\frac{C P U_{e x}}{C P U_{i m p}} \sim \frac{0.02}{\left(k \lambda_{D}\right)^{d}} \frac{c}{v_{A}} \min \left[\frac{1}{k \lambda_{D}}, \frac{c}{v_{A}} \sqrt{\frac{m_{e}}{m_{i}}}, \sqrt{\frac{m_{i}}{m_{e}}}\right] \frac{1}{N_{F E}},
$$

where $k=2 \pi / L, d_{e}$ is the electron skin depth, $m_{i}, m_{e}$ are the ion and electron masses, and $N_{F E}$ is the number of function (residual) evaluations (which indicates the number of repeated particle orbit computations). We see that the speedup increases with larger domain sizes (where $k \lambda_{D} \ll 1, d_{e} \gg \lambda_{D}$ ), and with $m_{i}$ much larger than $m_{e}$. Here, we vary the domain size $L$. It is expected that the cost of the implicit simulation will not change much with the domain size, provided that the number of cells and particles per cell are kept fixed, and the nonlinear iteration count is well controlled by the preconditioner. On the other hand, the explicit code is forced to maintain the same grid spacing and time step as the domain increases, to avoid numerical instability. Results are depicted in Fig. 8, and show good agreement with our simple estimate, 

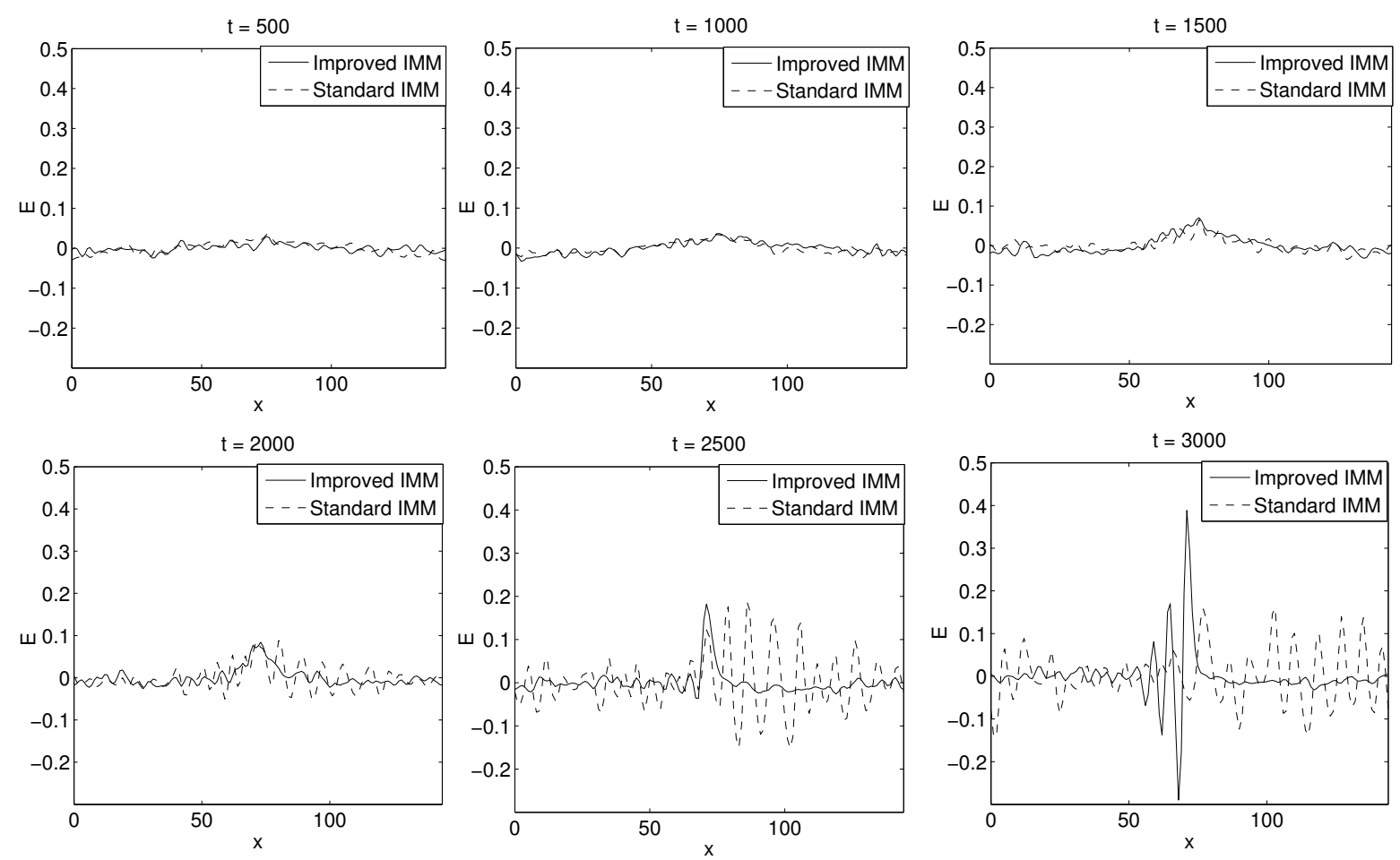

Figure 7: Electric field structure at various times for the Ion Acoustic Shock Wave test case

Eq. 40. In particular, it demonstrates that, for this 2D example, significant CPU speedups are possible for system sizes much larger than the Debye length $\left(>10^{4}\right.$ for $\left.k \lambda_{D}<10^{-3}\right)$.

\subsection{Collisional Vlasov-Fokker-Planck HOLO algorithms}

The dynamic evolution of coupled electromagnetic fields and collisional plasmas is described by the Vlasov-Fokker-Planck-Maxwell system. The Maxwell equations evolve electromagnetic fields based on moments of the ion-electron distribution functions (charge and current densities). The evolution of the distribution function for each species is governed by the Vlasov-Fokker-Planck (VFP) equation, which is coupled to the Maxwell system through electromagnetic forces. In this section, we will focus on an Eulerian approach which the VFP partial-differential equation is solved on a grid.

In the past, the Fokker-Planck equation has been used to model collisional dense plasmas for inertialconfinement-fusion conditions (ICF) [85, 86, 87, 88, 89, 90, 91]. In our work, we are motivated to consider implicit methods. A pioneering implicit study was carried out in reference [92], where a fully implicit total mass and energy conserving discretization of the Fokker-Planck operator in one velocity dimension (1V) was developed. In references [93,94], fully implicit, mass and energy conserving solver was developed for the 0D-2V Fokker-Planck equation. A fully implicit, 1D1V reduced Vlasov-Fokker-Planck-Quasi-NeutralField solver was developed in reference [95]. In the study in the reference, the collision operator was modeled using a reduced Fokker-Planck operator [96, 97] while solving for the field using the quasi-neutrality approximation. However, the method lacked discrete charge and energy conservation properties. Later, 


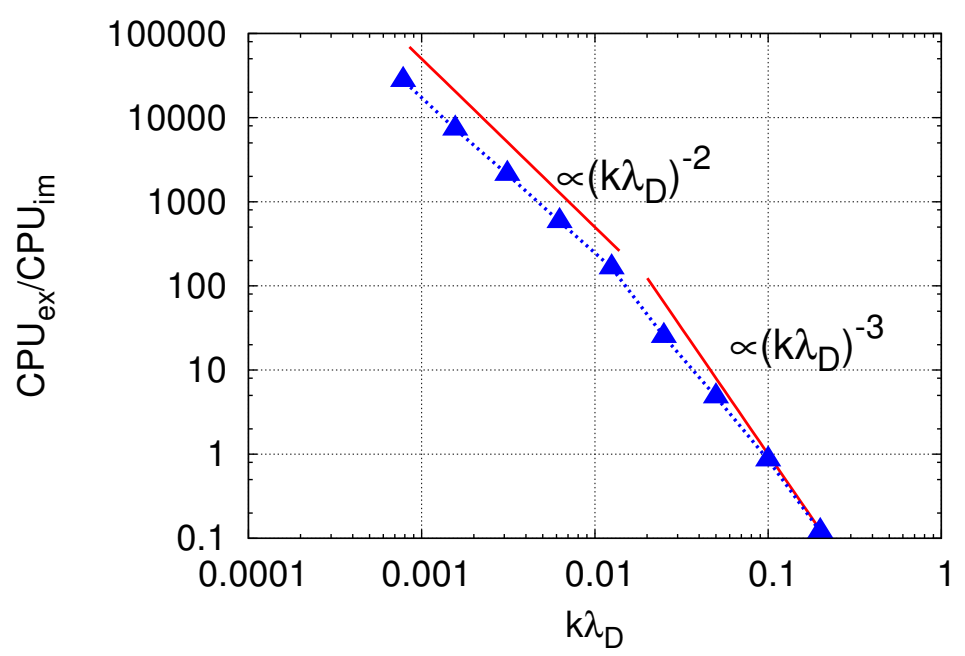

Figure 8: CPU speedup of implicit vs. explicit PIC for the electron Weibel instability case as a function of $k \lambda_{D} \sim \lambda_{D} / L$. Speedups of several orders of magnitude are possible for large domains $\left(\lambda_{D} / L \ll 1\right)$.

a fully implicit Vlasov-Maxwell solver was developed in [98], where the electrons were treated kinetically using a Cartesian tensor expansion [99] and ions were modeled as a cold fluid. For a more thorough recent review of numerical modeling of the Vlasov-Fokker-Planck system, see Ref. [100]. Finally, in the last few years, there have been significant developments on modern implicit solvers for the Fokker-Planck system $[101,102,103,104]$. In [101, 102] a charge and energy conserving 1D2V solver for the reduced Fokker-Planck system was developed, while in [103, 104] an adaptive, fully implicit, and fully mass, momentum, and energy conserving strategies were developed for a 0D2V full nonlinear Fokker-Planck collision operator.

We apply the HOLO algorithm to a coupled, electrostatic, 1D-1V, reduced Vlasov-Fokker-Planck-Ampère (VFPA) system with one translational degree of freedom. The VFP equation will be referred to as the HO system, and the moment-Ampère system will be referred to as the LO system. In the VFPA application, the $\mathrm{HO}$ system will provide the LO system with a self-consistent closure at the heat-flux level, while the LO system provides the HO system with the collision frequency, the collisional velocity space diffusion coefficient, the drag coefficients (both for the reduced Fokker-Planck operator), and the electric field. The method allows one to step over the inverse electron plasma frequency and electron collision time-scales.

\subsubsection{The Vlasov-Fokker-Planck model}

The 1D-1V Vlasov-Fokker-Planck equation for species $\alpha$ in the electrostatic limit is:

$$
\frac{\partial f_{\alpha}}{\partial t}+\mathbf{v} \cdot \nabla f_{\alpha}+\frac{q_{\alpha}}{m_{\alpha}} \mathbf{E} \cdot \nabla_{v} f_{\alpha}=\left(\frac{\partial f_{\alpha}}{\partial t}\right)_{c}
$$

with $\left(\frac{\partial f_{\alpha}}{\partial t}\right)_{c}$ the collision term, which is approximated here by a reduced Fokker-Planck operator [96, 97].:

$$
\left(\frac{\partial f_{\alpha}}{\partial t}\right)_{c}=\sum_{\beta}^{N_{s}} v_{\alpha \beta}\left\{D_{\alpha \beta} \frac{\partial^{2} f_{\alpha}}{\partial v^{2}}+\frac{\partial}{\partial v}\left[\left(v-u_{\beta}\right) f_{\alpha}\right]\right\}
$$


Together, they define the Vlasov-Fokker-Planck equation:

$$
\operatorname{VFP}_{\alpha}=\frac{\partial f_{\alpha}}{\partial t}+v \frac{\partial f_{\alpha}}{\partial x}+\frac{q_{\alpha}}{m_{\alpha}} E \frac{\partial f_{\alpha}}{\partial v}-\sum_{\beta}^{N_{s}} v_{\alpha \beta}\left\{D_{\alpha \beta} \frac{\partial^{2} f_{\alpha}}{\partial v^{2}}+\frac{\partial}{\partial v}\left[\left(v-u_{\beta}\right) f_{\alpha}\right]\right\}=0 .
$$

Here, $N_{s}$ is the total number of species in the system, $v_{\alpha \beta}=v_{\alpha \beta}(x, t)$ is the collision frequency for species $\alpha$ with species $\beta, D_{\alpha \beta}=D_{\alpha \beta}(x, t)$ is the collisional velocity space diffusion coefficient of species $\alpha$ with species $\beta$, and $u_{\beta}$ is the bulk fluid velocity of species $\beta . v_{\alpha \beta}$ and $D_{\alpha \beta}$ are both functions of the plasma species' density, $n$, and temperature, $T$.

The reduced Fokker-Planck operator [96, 97] features several key properties: 1) the equilibrium solution is a Maxwellian, 2) the velocity space diffusion and drag effects in the velocity space are captured, and 3) the operator conserves mass, momentum, and energy. The primary limitation of the reduced FokkerPlanck collision operator is the unphysically fast relaxation of energetic particles in a collisional time-scale, $v_{\alpha \beta}^{-1}$. This is to be contrasted with the true Fokker-Planck operator in terms of the Rosenbluth potentials [105], which relaxes the distribution function based on the relative velocities of the particles.

The electrostatic Ampère's equation reads:

$$
\epsilon_{0} \frac{\partial E}{\partial t}+\sum_{\alpha}^{N_{s}} q_{\alpha} n u_{\alpha}=0
$$

Here, $\epsilon_{0}$ is the permittivity of free space and $n u_{\alpha}$ is the number density flux of species $\alpha$,

$$
n u_{\alpha}=\left\langle v, f_{\alpha}\right\rangle_{v}
$$

Ampère's equation evolves the electric field in time to dynamically capture charge-separation fields. Equations 41 and 42 together define the Vlasov-Fokker-Planck-Ampère (VFPA) system. The system evolves the species distribution function and the field self-consistently in phase-space and time.

The VFPA system supports a wide range of time-scales. These time-scales include the inverse electron plasma frequency, $\omega_{p, e}^{-1}=\sqrt{m_{e} \epsilon_{0} / n_{e} q_{e}^{2}}$, like-species collision time-scale, $\tau_{\alpha \alpha}=v_{\alpha \alpha}^{-1}$, interspecies collision time-scale, $\tau_{\alpha \beta}=v_{\alpha \beta}^{-1}$, and slower dynamical time-scales. The time-scale disparity can span many orders of magnitude. For example, if we consider the implosion dynamics of an ICF capsule for Omega-facilityrelevant conditions [100], the longest time-scale is the capsule stagnation and peak compression, which is of order $10^{-9}$ [sec]. For the compressed core, the inverse electron plasma frequency is of order $10^{-18}$ [sec]. From the shortest time-scale of $\omega_{p_{e}}^{-1}$ to the longest physical time-scale of capsule achieving peak compression, more than nine orders of magnitude separation in physical time-scales exists.

In traditional explicit integration schemes, the time-step size, $\Delta t$, is limited by numerical stability constraints. For semi-implicit methods [106, 107], CFL conditions are relaxed and a larger $\Delta t$ can be chosen. However, if the solution is not iterated nonlinearly, large errors may accumulate from time-step to timestep. If one wishes to iterate the Vlasov-Ampère system to convergence using a standard Picard iteration, it can be demonstrated that, for the method to converge, the time-step size must resolve the inverse plasma 
frequency, $\omega_{p, e}^{-1}[108]$. If the gradient scale-length of the solution is not comparable to the Debye length, $\lambda_{D}$, and the dynamical time-scale of the problem is longer than $\omega_{p, e}^{-1}$, then the VFPA system becomes stiff. In the next section, we present an effective nonlinear solver strategy based on the HOLO acceleration scheme to effectively step over such stiff time scales.

\subsubsection{HOLO VFP algorithm}

To solve the VFPA system nonlinearly, an implicit evaluation of the collisional coefficients $\left(D_{\alpha \beta}, u_{\alpha \beta}\right.$, and $\left.v_{\alpha \beta}\right)$, field, and distribution functions is required. In our implementation, the HOLO algorithm accelerates the convergence of the standard Picard iteration by using the LO moment and field equation to provide estimates for these coefficients and the electric field. In turn the $\mathrm{HO}$ (kinetic) system will provide the closure quantities for the LO system such as the stress tensor and the heat flux.

To derive the corresponding LO moment equations, we take the $1, v$, and $\frac{v^{2}}{2}$ moments of the VFP equation to obtain the continuity equation,

$$
\left\langle 1, \operatorname{VFP}_{\alpha}\right\rangle_{v}=\frac{\partial n_{\alpha}}{\partial t}+\frac{\partial}{\partial x} n u_{\alpha}=0
$$

the momentum equation,

$$
\left\langle v, \operatorname{VFP}_{\alpha}\right\rangle_{v}=\frac{\partial n u_{\alpha}}{\partial t}+\frac{\partial}{\partial x}\left[n u_{\alpha}^{2}+\frac{P_{\alpha}}{m_{\alpha}}\right]-\frac{q_{\alpha}}{m_{\alpha}} n_{\alpha} E+\sum_{\beta \neq \alpha}^{N_{s}} v_{\alpha \beta} n_{\alpha}\left(u_{\alpha}-u_{\beta}\right)=0,
$$

and the energy equation,

$$
\begin{array}{r}
\frac{1}{2}\left\langle v^{2}, \operatorname{VFP}_{\alpha}\right\rangle_{v}=\frac{\partial U_{\alpha}}{\partial t}+\frac{\partial}{\partial x} \\
{\left[u_{\alpha}\left(U_{\alpha}+\frac{P_{\alpha}}{m_{\alpha}}\right)+\frac{Q_{\alpha}}{m_{\alpha}}\right]-\frac{q_{\alpha}}{m_{\alpha}} n u_{\alpha} E-} \\
\sum_{\beta \neq \alpha}^{N_{s}} v_{\alpha \beta}\left\{D_{\alpha \beta} n_{\alpha}-\left[S_{2, \alpha}-n u_{\alpha} u_{\beta}\right]\right\}=0 .
\end{array}
$$

Here, $P_{\alpha}$ is the scalar pressure,

$$
P_{\alpha}=m_{\alpha}\left\langle\left(v-u_{\alpha}\right)^{2}, f_{\alpha}\right\rangle_{v}=n_{\alpha} T_{\alpha}=2 m_{\alpha}\left[U_{\alpha}-0.5\left(n u_{\alpha}\right)^{2} / n_{\alpha}\right],
$$

$Q_{\alpha}$ is the heat-flux,

$$
Q_{\alpha}=\frac{m_{\alpha}}{2}\left\langle\left(v-u_{\alpha}\right)^{3}, f_{\alpha}\right\rangle_{v}
$$

$m_{\alpha} U_{\alpha}$ is the total energy density

$$
m_{\alpha} U_{\alpha}=\frac{m_{\alpha}}{2}\left\langle v^{2}, f_{\alpha}\right\rangle_{v}
$$

and $m_{\alpha} S_{2, \alpha}$ is the total stress tensor,

$$
m_{\alpha} S_{2, \alpha}=m_{\alpha}\left\langle v^{2}, f_{\alpha}\right\rangle_{v}=n_{\alpha} T_{\alpha}+m_{\alpha}\left(n u_{\alpha}\right)^{2} / n_{\alpha}
$$

We retain the $2^{\text {nd }}$ moment equation for the temperature, $T_{\alpha}$, to evaluate for $S_{2, \alpha}$. This is not the case for 
the moment-based acceleration algorithm for a collisionless plasma discussed in Refs. [13] and [101]. We note that, for one translational degree of freedom, the viscous stress evaluates to zero and only the scalar pressure survives [58].

As in earlier applications considered in this paper, we employ consistency terms to ensure discrete consistency between $\mathrm{HO}$ and LO descriptions. The LO system with consistency terms $\gamma^{\mathrm{HO}}$ and $\eta^{\mathrm{HO}}$ reads [102]:

$$
\begin{gathered}
\frac{\partial n_{\alpha}^{L O}}{\partial t}+\frac{\partial}{\partial x} n u_{\alpha}^{L O}-\frac{\partial \gamma_{n, \alpha}^{H O}}{\partial x}-\eta_{n, \alpha}^{H O}=0 \\
\frac{\partial n u_{\alpha}^{L O}}{\partial t}+\frac{\partial}{\partial x}\left[\left(n u^{L O}\right)_{\alpha}^{2}+\frac{P_{\alpha}^{L O}}{m_{\alpha}}\right]-\frac{q_{\alpha}}{m_{\alpha}} n_{\alpha}^{L O} E+\sum_{\beta \neq \alpha}^{N_{s}} v_{\alpha \beta}^{L O} n_{\alpha}^{L O}\left(u_{\alpha}^{L O}-u_{\beta}^{L O}\right)-\frac{\partial \gamma_{n u, \alpha}^{H O}}{\partial x}-\eta_{n u, \alpha}^{H O}=0 \\
\frac{\partial U_{\alpha}^{L O}}{\partial t}+\frac{\partial}{\partial x}\left[u_{\alpha}^{L O}\left(U_{\alpha}^{L O}+\frac{P_{\alpha}^{L O}}{m_{\alpha}}\right)+\frac{Q_{\alpha}^{L O}}{m_{\alpha}}\right]-\frac{q_{\alpha}}{m_{\alpha}} n u_{\alpha}^{L O} E- \\
\sum_{\beta \neq \alpha}^{N_{s}} v_{\alpha \beta}^{L O}\left\{D_{\alpha \beta}^{L O} n_{\alpha}^{L O}-\left[S_{2, \alpha}^{L O}-n u_{\alpha}^{L O} u_{\beta}\right]\right\}-\frac{\partial \gamma_{U, \alpha}^{H O}}{\partial x}-\eta_{U, \alpha}^{H O}=0 .
\end{gathered}
$$

The coupling with Ampère's equation completes the formulation of the LO system:

$$
\epsilon_{0} \frac{\partial E}{\partial t}+\sum_{\alpha}^{N_{s}} q_{\alpha} n u_{\alpha}^{L O}=0
$$

We point out that, unlike the gas kinetics application [17], the heat flux in Eq. 53 is not evaluated from the HO solution. Rather, to capture the stiffness associated with the electron thermal conduction time-scale, we use the Braginskii closure,

$$
Q_{\alpha}^{L O}=-\kappa_{\alpha}^{L O} \frac{\partial T_{\alpha}^{L O}}{\partial x}
$$

where $\kappa_{\alpha}$ is the Braginskii thermal conductivity for species $\alpha$. The Braginskii closure is appropriate for sufficiently collisional plasmas [109]. For weakly collisional plasmas, the kinetic behavior in the heat flux will be captured by the corresponding consistency terms in Eq. 53 (recall that consistency terms ensure that $\mathrm{HO}$ and LO solutions are consistent upon convergence). More details are provided in Ref. [102].

The HO formulation is nonlinearly eliminated from the LO nonlinear residual via the discrete consistency terms. Once a LO solution state is available, it can be used to provide collisional coefficients and the electric field to the HO system, which can then be solved for the distribution function $f_{\alpha}$ from:

$$
\frac{\partial f_{\alpha}}{\partial t}+v \frac{\partial f_{\alpha}}{\partial x}+\frac{q_{\alpha}}{m_{\alpha}} E \frac{\partial f_{\alpha}}{\partial v}-\sum_{\beta}^{N_{s}} v_{\alpha \beta}^{L O}\left\{D_{\alpha \beta}^{L O} \frac{\partial^{2} f_{\alpha}}{\partial v^{2}}+\frac{\partial}{\partial v}\left[\left(v-u_{\beta}^{L O}\right) f_{\alpha}\right]\right\}=0 .
$$

Once $f_{\alpha}$ is found, it can be used to compute the consistency terms in the LO system, which can then be solved to update the LO solution state.

\subsubsection{Numerical results}

We demonstrate the performance of the new VFPA HOLO solver on two model problems: 1) a homogeneous $0 \mathrm{D} 1 \mathrm{~V}$ thermal equilibration test between electrons and a single ion species, and 2) a two-species 
Table 5: Comparison in performance of the new HOLO algorithm and a standard Picard iteration for varying $\Delta t$.

\begin{tabular}{lccc}
\hline & $\Delta t=1 \tau_{e e}$ & $\Delta t=10 \tau_{e e}$ & $\Delta t=100 \tau_{e e}$ \\
\hline \hline Iteration $_{\text {HOLO }}$ & 2 & 2 & 4 \\
Iteration $_{\text {Picard }}$ & 12 & 94 & 865 \\
\hline
\end{tabular}

collisional ion-acoustic-wave problem. The purpose of the simulations is to demonstrate the algorithmic performance of the new HOLO algorithm for the VFPA system.

Two-Species Thermal Equilibration Problem. We begin to test the proposed algorithm with a 0D1V two-species thermal equilibration problem. We consider an electron and proton plasma. We show that the new algorithm allows one to use a time-step size, $\Delta t$, much larger than the electron-electron collision time-scale, $\tau_{e e}$, while following the electron-ion thermalization time-scale, $\tau_{i e}$, accurately. We show that the new algorithm allows one to nonlinearly converge the coupled Fokker-Planck equation with electrons and protons efficiently compared to a standard Picard iteration scheme.

We initialize the problem with an electron temperature $T_{e}=1.1$ and a proton ion temperature $T_{i}=0.9$. For this test problem, we assume a constant collision time-scale of $\tau_{e e}=v_{e e}^{-1}=1 / 60.5=0.0165, \tau_{i i}=v_{i i}^{-1}=$ 1 , and a thermal relaxation time-scale of, $\tau_{i e}=v_{i e}^{-1}=1836 / 60.5=30.29$. We numerically solve the 0D1V reduced Fokker-Planck problem,

$$
\frac{\partial f_{\alpha}}{\partial t}=\sum_{\beta}^{N_{s}} v_{\alpha \beta}\left\{D_{\alpha \beta} \frac{\partial^{2} f_{\alpha}}{\partial v^{2}}+\frac{\partial}{\partial v}\left[\left(v-u_{\beta}\right) f_{\alpha}\right]\right\}
$$

In Table 5, we compare the performance of the new HOLO algorithm and a standard Picard iteration applied to the two-species thermal equilibration problem for time-step sizes of $\Delta t=1 \tau_{e e}, 10 \tau_{e e}$, and $100 \tau_{e e}$. As can be seen, the performance of the new HOLO algorithm is one to several orders-of-magnitude superior to the standard Picard iteration for all regimes of $\Delta t$. We note that the cost of the LO solver relative to the $\mathrm{HO}$ solver is negligible.

Two-Species Collisional Ion-Acoustic-Wave Problem. We test the new algorithm on a 1D1V, two-species collisional ion-acoustic-wave (IAW) problem. The purpose of this problem is to demonstrate the algorithmic performance as a function of $\Delta t$ and demonstrate that we can step over both the inverse electron plasma frequency and the electron collision time-scales. We consider electron and proton with a mass of $m_{e}=1 / 1836$ and $m_{i}=1$, respectively, and an initial temperature of $T_{e, 0}=T_{i, 0}=1$. The problem is initialized using a sinusoidal perturbation in the density and fluid velocity,

$$
\begin{gathered}
n_{\alpha, 0}(x, t=0)=1+0.2 \sin k_{x} x, \\
u_{\alpha, 0}(x, t=0)=0.2 \sin k_{x} x
\end{gathered}
$$


Table 6: Number of Picard iterations with varying $\Delta t$ and $N$ for a collisional IAW problem.

\begin{tabular}{cccc}
\hline$\Delta t$ & $N=50$ & $N=100$ & $N=200$ \\
\hline \hline $0.1 \omega_{p, e}^{*,-1}$ & 2 & 2 & 2 \\
$1 \omega_{p, e}^{*,-1}$ & 6 & 6 & 6 \\
$2 \omega_{p, e}^{*,-1}$ & diverged & diverged & diverged \\
\hline
\end{tabular}

Table 7: Number of HOLO iterations with varying $\Delta t$ and $N$ for a collisional IAW problem

\begin{tabular}{cccc}
\hline$\Delta t$ & $N=50$ & $N=100$ & $N=200$ \\
\hline \hline $2 \omega_{p, e}^{*,-1}$ & 3 & 2 & 2 \\
$20 \omega_{p, e}^{*,-1}$ & 6 & 5 & 5 \\
$200 \omega_{p, e}^{*,-1}$ & 10 & 9 & 7 \\
$1000 \omega_{p, e}^{*,-1}$ & 17 & 12 & 12 \\
\hline
\end{tabular}

where $k_{x}=2 \pi / L_{x}$ is the wave vector of the perturbation, and $L_{x}=200$ is the system length and the spatial boundaries are periodic. In the study, we consider several spatial grid resolutions, $N=50,100$, and 200. The initial distribution function is assumed to be a Maxwellian,

$$
f_{\alpha}(x, v, t=0)=\frac{n_{\alpha, 0}}{\sqrt{2 \pi T_{\alpha, 0} / m_{\alpha}}} \exp \left[-\frac{m_{\alpha}\left(v-u_{\alpha, 0}\right)^{2}}{2 T_{\alpha, 0}}\right] .
$$

We compare the performance difference between a standard Picard iteration scheme and the HOLO algorithm. First, we demonstrate that the standard Picard iteration algorithm is divergent when $\Delta t>\omega_{p, e}^{*,-1}$. In Tables 6 and 7, the number of Picard and HOLO iterations required to converge the coupled VFPA system are shown for different $\Delta t$ and $N$. It can be seen that, for $\Delta t=2 \omega_{p, e}^{*,-1}$, the Picard iteration scheme diverges. However, the number of HOLO iterations grows only very slightly with increasing $\Delta t$, and $\Delta t$ can be much larger than that of the standard Picard iteration scheme.

\section{Application 4: Ocean modeling}

Accurate modeling of ocean circulation under various forcings is of great importance, and impacts a variety of political decisions. In particular, it has been shown that high resolution is necessary for an accurate simulation of long-time ocean thermohaline circulation [110]. Scientists are currently performing ocean simulations with semi-implicit [9] and split-explicit methods [111, 112]. Typically, these methods utilize a barotropic-baroclinic splitting that isolates fast (barotropic) and slow (baroclinic) time scales. The faster external gravity waves (or barotropic motions) are independent of depth, and thus two dimensional, while the slower baroclinic motions are fully three dimensional. For most problems of interest, explicit time discretizations are impractical for these systems, due to short timesteps imposed by the fast waves.

Methods based on barotropic-baroclinic splitting can easily be cast in the framework of HOLO methods. Indeed, the semi-implicit (SI) method utilized in the Parallel Ocean Program (POP) $[9,40]$ and splitexplicit (SE) methods utilized in Model for Prediction Across Scales (MPAS) [113] and Modular Ocean Model (MOM) [114] are characterized by a LO barotropic system obtained by vertical moment of the HO 
baroclinic continuity and momentum equations.

In this section, we propose a multirate HOLO method [115, 116] (following similar ideas as proposed in Refs. $[117,118,119])$ based on the barotropic-baroclinic decomposition. The two-dimensional barotropic scalar continuity equation is treated implicitly with a preconditioned Jacobian-free Newton-Krylov method (JFNK). The baroclinic three-dimensional equations are nonlinearly eliminated from the main JFNK residual, and subcycled explicitly. In this approach, the memory footprint of the underlying Krylov vector is greatly reduced over that required by fully coupled implicit methods. The method is second-order accurate and scales algorithmically, with allowed timesteps much larger than fully explicit methods. Moreover, the hierarchical nature of the algorithm lends itself readily to emerging architectures. In particular, we introduce a communication staging strategy $[115,116]$ for the three dimensional explicit system that greatly reduces the communication costs of the algorithm and provides a key advantage as communication costs continue to dominate relative to floating point costs in emerging architectures.

\section{1. z-level ocean model}

We assume a z-level ocean model in Cartesian coordinates, in $K$ layers, where $k=1$ corresponds to the top layer [9]. The momentum and continuity equations are given by

$$
\begin{gathered}
\frac{\partial \mathbf{u}_{k}}{\partial t}+L_{1}\left(\mathbf{u}_{k}, \eta\right)+f \mathbf{u}_{k}^{\perp}+G_{1}\left(\varphi_{i, k}, \eta\right)+G_{2}\left(\varphi_{i, k}\right)=0, \quad k=1, \ldots, K, \\
\frac{\partial \eta}{\partial t}+D\left(\mathbf{u}_{1}, \eta\right)=0, \quad k=1, \quad \text { and } \\
\nabla \cdot\left(h_{k}(\eta) \mathbf{u}_{k}\right)+w_{k-1 / 2}-w_{k+1 / 2}=0, \quad k=2, \ldots, K,
\end{gathered}
$$

with

$$
\begin{gathered}
L_{1}\left(\mathbf{u}_{k}, \eta\right)=\left(\mathbf{u}_{k} \cdot \nabla\right) \mathbf{u}_{k}+\frac{w_{k-1 / 2} \mathbf{u}_{k-1 / 2}-w_{k+1 / 2} \mathbf{u}_{k+1 / 2}}{h_{k}(\eta)}, \\
G_{1}\left(\varphi_{i, k}, \eta\right)=\frac{1}{\rho_{0}} g \nabla \rho_{1}\left(\varphi_{i, k}\right) \eta \\
G_{2}\left(\varphi_{i, k}\right)=\frac{1}{\rho_{0}} \nabla p_{H k}\left(\varphi_{i, k}\right) \text { and } \\
D\left(\mathbf{u}_{1}, \eta\right)=\nabla \cdot\left(h_{1}(\eta) \mathbf{u}_{1}\right)-w_{3 / 2}
\end{gathered}
$$

where the vertical discretization consists of equally spaced layers of thickness $\Delta z$, and $\mathbf{u}_{k}=\left[u_{k} v_{k}\right]^{\mathrm{T}}$ is the horizontal velocity at mid-layer $k$, the horizontal velocity at the top and bottom of layer $k$ are given by $\mathbf{u}_{k-1 / 2}$ and $\mathbf{u}_{k+1 / 2}$ with $\mathbf{u}_{k \pm 1 / 2}=\left(\mathbf{u}_{k}+\mathbf{u}_{k \pm 1}\right) / 2$. The pressure at the bottom of layer $k$ is given by $p_{k}$, $w_{k-1 / 2}$ is the vertical velocity at the top of layer $k, w_{k}$ is the vertical velocity at mid-layer $k, \eta$ is sea surface height perturbation, $h_{k}$ is the thickness of layer $k$ with $h_{k}=\Delta z_{k}$ for $k \neq 1$ and $h_{1}=\Delta z_{1}+\eta$, $f$ is the Coriolis parameter, $\mathbf{u}_{k}^{\perp}=\left[-v_{k} u_{k}\right]^{\mathrm{T}}, \rho_{0}=1000$ is the reference density, $t$ corresponds to time, and $\nabla$ and $\nabla$. are the gradient and divergence operators in the horizontal plane. We augment Eqs. 61-63 with transport 
equations for temperature $\varphi_{1, k}$, and salinity $\varphi_{2, k}$ in each layer $k$ :

$$
\frac{\partial}{\partial t}\left(h_{k}(\eta) \varphi_{i, k}\right)+L_{2}\left(\varphi_{i, k}, \mathbf{u}_{k}, \eta\right)=0, \quad i=1,2
$$

with

$$
L_{2}\left(\varphi_{i, k}, \mathbf{u}_{k}, \eta\right)=\nabla \cdot\left(h_{k}(\eta) \varphi_{i, k} \mathbf{u}_{k}\right)+\frac{w_{k-1 / 2} h_{k-1 / 2}(\eta) \varphi_{i, k-1 / 2}-w_{k+1 / 2} h_{k+1 / 2}(\eta) \varphi_{i, k+1 / 2}}{h_{k}(\eta)}
$$

and a linear equation of state for density given by

$$
\rho_{k}\left(\varphi_{1, k}, \varphi_{2, k}\right)=\rho_{0}\left(1.0-\alpha \varphi_{1, k}+\beta \varphi_{2, k}\right),
$$

with $\alpha=2.5 \times 10^{-4}$ and $\beta=7.6 \times 10^{-4}$ [40]. The pressure at the bottom of layer $k$ is given by

$$
p_{k}=g \rho_{1} \eta+\sum_{l=1}^{k} g \rho_{l} h_{l}=g \rho_{1} \eta+p_{H k}
$$

where $g$ is acceleration due to gravity and $p_{H k}$ is hydrostatic pressure at the bottom of layer $k$. Due to Eq. $70, p_{k}=p_{k}\left(\varphi_{i, k}\right)$.

\subsection{The HOLO ocean algorithm}

The fastest time scale associated with Eqs. $61-63$ and 68 is the barotropic timescale, due to the external gravity-wave, $\Delta t_{g}=\Delta x(g D)^{-1 / 2}$, with speed on order of $200 \mathrm{~m} / \mathrm{s}$. The baroclinic timescale, $\Delta t_{a}$, is due to advection or dynamic time scales with speed on order of $10 \mathrm{~m} / \mathrm{s}$, and is therefore much slower. The separation between these time scales presents difficulties for time integration. Explicit methods are generally unstable for timesteps larger than the external gravity-wave time scale.

To obtain the LO system, we introduce the barotropic vertical-averaging operator:

$$
\overline{\mathbf{u}}=\frac{1}{H(\eta)} \sum_{k=1}^{K} h_{k}(\eta) \mathbf{u}_{k}
$$

where $H(\eta)=\sum_{k=1}^{K} h_{k}(\eta)$ and $\overline{\mathbf{u}}$ is the barotropic velocity obtained by the vertical average of the velocity. Application of the barotropic operator Eq. 72 to Eqs. 61-63 yields the LO problem:

$$
F(\eta)=\frac{\partial \eta}{\partial t}+\nabla \cdot H(\eta) \overline{\mathbf{u}}(\eta)=0
$$

and the $\mathrm{HO}$ problem is given by Eqs. 61-63 and 68. Equation 73 is discretized in time using trapezoidal rule [120] with timestep $\Delta t_{\mathrm{LO}}$, and solved by a preconditioned JFNK method each timestep. We utilize the preconditioner developed in [121]. In order to evaluate Eq. 73, we need to obtain the term $H(\eta) \overline{\mathbf{u}}(\eta)$ from the $\mathrm{HO}$ problem, Eqs. 61-63. The HO problem is discretized in time in a multirate fashion, via explicit subcycling with a predictor-corrector method, and enslaved to the LO problem, Eq. 73 [115, 116]. The subcycling process is executed for every linear iteration, and thus effective preconditioning is vital to minimize the total number of function evaluations per LO timestep. 


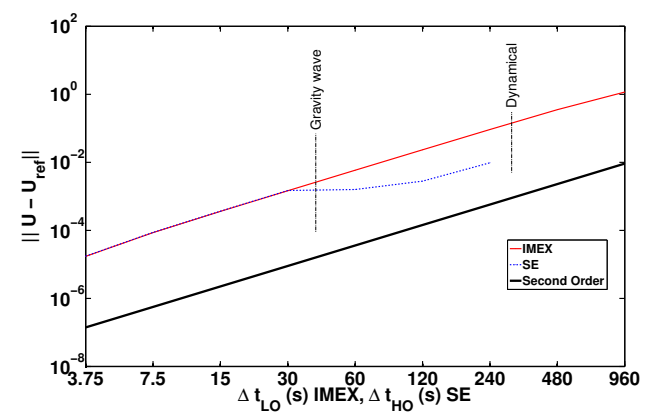

(a)

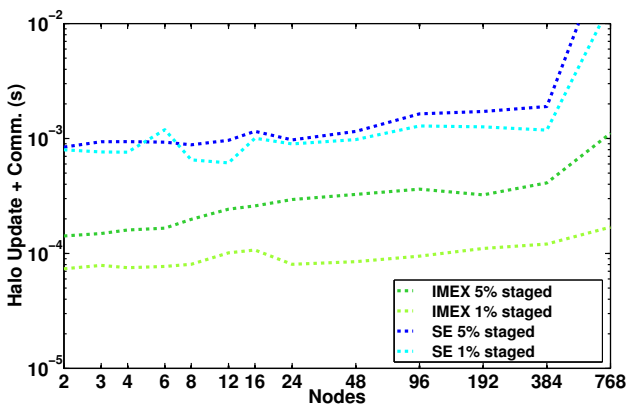

(c)

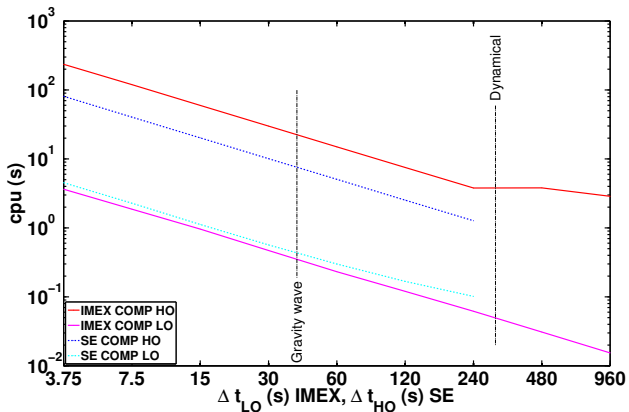

(b)

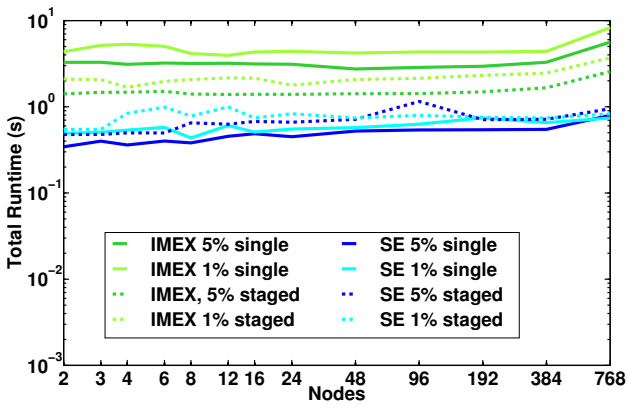

(d)

Figure 9: (a) Timestep size vs. error for IMEX and SE. Error rate is computed relative to a reference solution $U_{\text {ref }}$ obtained by fourthorder RK4 with $\Delta t=1.875$. (b) Timestep size vs. CPU for HO and LO components of IMEX and SE. (c) IMEX with $\Delta t_{\mathrm{LO}}=900$ $\left(\Delta t_{\mathrm{HO}}=\Delta t_{d}\right)$, and SE with $\Delta t_{\mathrm{HO}}=960\left(\Delta t_{\mathrm{LO}}=\Delta t_{g}\right), 1-768$ nodes. (d) Total runtime per degree of freedom for IMEX with $\Delta t_{\mathrm{LO}}=900\left(\Delta t_{\mathrm{HO}}=\Delta t_{d}\right)$, and SE with $\Delta t_{\mathrm{HO}}=900\left(\Delta t_{\mathrm{LO}}=\Delta t_{g}\right), 1-768$ nodes.

\subsection{Numerical results}

We present a numerical example to demonstrate convergence of the multirate HOLO method (termed IMEX below), and to compare serial and parallel performance between IMEX and the split-explicit (SE [113]) method.

The domain consists of a two dimensional infinite channel and is discretized horizontally with a C-grid finite volume scheme [122] with $\Delta x=1.0 \times 10^{4}, K=60, L=5.0 \times 10^{6}, D=5.0 \times 10^{3}, f=1.0 \times 10^{-4}$. The fluid is initially at rest, with an initial perturbation in $\eta$. In addition, the initial condition for temperature is a linear distribution in depth with $\varphi_{1,1}=20.0$ and $\varphi_{1,60}=10.0$, combined with a perturbation in layers near the surface. The initial condition for salinity is a linear distribution in depth with $\varphi_{2,1}=30.0$ and $\varphi_{2,60}=26.0$. Subcycling of the $\mathrm{HO}$ component is required such that $\Delta t_{\mathrm{HO}}=\Delta t_{a}$ when $\Delta t_{\mathrm{LO}}>\Delta t_{a}$, and subcycling of the LO component of SE is required such that $\Delta t_{\mathrm{LO}}=\Delta t_{g}$ when $\Delta t_{\mathrm{HO}}>\Delta t_{g}$.

Fig. 9a shows error as a function of timestep size for IMEX (solid line) and SE (dotted line) and clearly shows IMEX and SE to have second-order convergence. Note that SE shows a transition zone between second-order slopes, which occurs at timesteps where subcycling becomes active. The transition reflects the fact that the error is dominated by the smaller (subcycling) timestep. Fig. $9 \mathrm{~b}$ shows total serial CPU time (both HO and LO components) as a function of timestep size for IMEX and SE, and shows both methods scale well, although IMEX costs more than SE over the range of timesteps. Note that the LO SE component 
costs more and does not scale as well as the IMEX LO; and HO is the dominant cost in both SE and IMEX. The algorithmic scability of the IMEX LO problem is achieved entirely by effective preconditioning. These results show that the IMEX algorithm is algorithmically scalable and second-order accurate, with serial performance comparable to the split-explicit method. Note that in Figs. 9a-9b, the timestep axis refers to the outer timestep: $\Delta t_{\mathrm{LO}}$ for IMEX and $\Delta t_{\mathrm{HO}}$ for SE.

When IMEX is implemented with an effective communication staging strategy, cost of the IMEX HO solver in parallel can be significantly reduced. Most ocean models utilize domain decomposition in the horizontal surface, with subdomains extruded vertically in depth. Thus, there is horizontal halo exchange (communication) required on each layer. Traditional single-halo implementations perform a single halo exchange once per timestep or stage. Our communication staging strategy [115] aggregates the network communications of halo cells necessary for multiple explicit updates by increasing the width of the halo band relative to the number of staged timesteps. In our implementation, the halo width is increased and the necessary halo communication for multiple timesteps is performed in advance at the beginning of the timestep, followed by subcycled timesteps. This strategy particularly favors our IMEX method over SE, due to the fact that IMEX subcycles the HO components over longer timesteps, rather than subcycling the LO components over smaller timesteps.

Figs. 9c-9d show weak scaling results to evaluate effectiveness of the communication staging strategy for both IMEX and SE, with $\Delta t_{\mathrm{HO}}=900\left(\Delta t_{\mathrm{LO}}=\Delta t_{g}\right)$ for SE and $\Delta t_{\mathrm{LO}}=900\left(\Delta t_{\mathrm{HO}}=\Delta t_{a}\right)$ for IMEX. With this choice of outer timestep size, the SE LO component must be subcycled 30 times and the IMEX $\mathrm{HO}$ component must be subcycled 3 times. The motivation for this problem is to compare the relative communication cost for IMEX and SE for integration at large timesteps. The computations were performed on the Mustang system at Los Alamos National Laboratory. To demonstrate network communication effects, these examples were run using one single-threaded MPI rank per node (one compute-core per node) on 1-768 nodes in two weak scaling configurations. The first configuration, denoted $5 \%$ fixes the ratio of halo width to local vector length to $1: 9$, and the second configuration, denoted $1 \%$, fixes the ratio to $1: 49$. Fig. $9 \mathrm{c}$ shows that the communication staging strategy reduces the relative communication costs for IMEX by an order of magnitude over that of SE.

Fig. $9 \mathrm{~d}$ shows the total computation time per degree of freedom for SE with $\Delta t_{\text {но }}=900\left(\Delta t_{\mathrm{LO}}=\Delta t_{g}\right)$ and IMEX with $\Delta t_{\mathrm{LO}}=900\left(\Delta t_{\mathrm{HO}}=\Delta t_{d}\right), 1-768$ nodes, single-halo and staged communication. The figure confirms the weak scaling nature of the test, with computation rapidly becoming a fixed cost per method, choice of staged versus single-halo, and type of halo size. Fig. 9d also shows that staging increased the computational time for SE, while reducing that of IMEX.

These results show that, with an effective communication staging strategy and subcycling of the $\mathrm{HO}$ problem, our IMEX method in a parallel environment can decrease the relative communication by more than two orders of magnitude over the split-explicit approach. This makes IMEX ideally suited for heterogeneous massively-parallel environments, where communication costs continue to grow and are dominant 
relative to floating-point costs. The increase in savings of relative communication costs of the IMEX method over SE in the regime of large timesteps and large number of nodes is made possible by the ability to subcycle the HO component within an implicit treatment of the LO component. While our results have been obtained in 2D, the analysis in [116] suggests similar savings in relative communication costs in 3D.

\section{Conclusions}

We have presented a broad view of the state of the art in modern HOLO algorithms and their application to challenging multiscale problems, with examples in radiation transport, gas dynamics, plasmas, and ocean modeling. Across this broad set of applications, HOLO algorithms have demonstrated to be either more efficient or more accurate (or both) than conventional approaches, underscoring their potential for multiscale simulations, and their readiness to play a crucial role in high-fidelity multiscale computing at the exascale.

\section{Acknowledgments}

This work was partially sponsored by the Los Alamos National Laboratory Directed Research and Development (LDRD) program, by the Los Alamos National Laboratory Advance Simulation and Computing (ASC) program, and by the Office of Applied Scientific Computing Research (ASCR) and Office of Biological and Environmental Research (BER) of the US Department of Energy. This work was performed

under the auspices of the National Nuclear Security Administration of the U.S. Department of Energy at Los Alamos National Laboratory, managed by LANS, LLC under contract DE-AC52-06NA25396. 
[1] V. Y. Gol'din, "A quasi-diffusion method of solving the kinetic equation," USSR Computational Mathematics and Mathematical Physics, vol. 4, pp. 136-149, 1967.

[2] R. E. Alcouffe, “Diffusion synthetic acceleration methods for the diamond differenced discrete ordinates equations," Nuclear Science and Engineering, vol. 66, pp. 344-355, 1977.

[3] K.-H. A. Winkler, M. L. Norman, and D. Mihalas, Implicit adaptive-grid radiation hydrodynamics, pp. 145-184. Dec. 1985.

[4] E. Larsen, "A grey transport acceleration method for time-dependent radiative transfer problems," Journal of Computational Physics, vol. 78, pp. 459-480, 1988.

[5] R. J. Mason, "Implicit moment particle simulation of plasmas," J. Comput. Phys., vol. 41, no. 2, pp. 233 $-244,1981$.

[6] J. Denavit, “Time-filtering particle simulations with $\omega_{p e} \Delta t \gg 1$," J. Comput. Phys., vol. 42, no. 2, pp. $337-366,1981$.

[7] J. U. Brackbill and D. W. Forslund, "An implicit method for electromagnetic plasma simulation in two dimensions," J. Comput. Phys., vol. 46, p. 271, 1982.

[8] J. Brackbill and D. Forslund, "Simulation of low-frequency electromagnetic phenomena in plasmas," in Multiple time scales (J. U. Brackbill and B. I. Cohen, eds.), Academic Press, 1985.

[9] J. Dukowicz and R. Smith, "Implicit free-surface method for the Bryan-Cox-Semtner ocean model," J. Geophys. Res., vol. 99, no. C4, pp. 7991-8014, 1994.

[10] P. Lanzdron, D. Rose, and J. Wilkes, "An analysis of approximate nonlinear elimination," SIAM Journal on Scientific Computing, vol. 17, pp. 538-559, 1996.

[11] H. X. Vu and J. U. Brackbill, "CELEST1D: an implicit, fully kinetic model for low-frequency, electromagnetic plasma simulation," Comput. Phys. Commun., vol. 69, pp. 253-276, 1992.

[12] G. Lapenta and J. Brackbill, “CELESTE 3D: Implicit adaptive grid plasma simulation,” in International School/Symposium for Space Simulation, (Kyoto, Japan), March 13-19 1997.

[13] W. T. Taitano, D. A. Knoll, L. Chacón, and G. Chen, "Development of a consistent and stable fully implicit moment method for vlasov-ampère particle in cell (pic) system," SIAM Journal on Scientific Computing, vol. 35, no. 5, pp. S126-S149, 2013.

[14] D. Knoll, H. Park, and K. Smith, "Application of the Jacobian-free Newton-Krylov method to nonlinear acceleration of transport source iteration in slab geometry," Nuclear Science and Engineering, vol. 167, pp. 122-132, 2011. 
[15] H. Park, D. A. Knoll, and C. K. Newman, "Nonlinear acceleration of transport criticality problem," Nuclear Science and Engineering, vol. 172, pp. 52-65, 2012.

[16] H. Park, D. A. Knoll, R. M. Rauenzahn, A. B. Wollaber, and J. D. Densmore, “A consistent, momentbased, multiscale solution approach for thermal radiative transfer problems," Transport Theory and Statistical Physics, vol. 41, pp. 284-303, 2012.

[17] W. Taitano, D. Knoll, L. Chacón, J. Reisner, and A. Prinja, “Moment-based acceleration for neutral gas kinetics with bgk collision operator," J. Comp. and Theo. Transp., vol. 43, no. 1-7, pp. 83-108, 2014.

[18] G. Chen, L. Chacón, C. A. Leibs, D. A. Knoll, and W. Taitano, "Fluid preconditioning for NewtonKrylov-based, fully implicit, electrostatic particle-in-cell simulations," Journal of computational physics, vol. 258, p. 555, 2014.

[19] G. Chen and L. Chacón, "An energy- and charge-conserving, nonlinearly implicit, electromagnetic 1D-3V Vlasov-Darwin particle-in-cell algorithm," Comput. Phys. Commun., vol. 185, no. 10, pp. 2391$2402,2014$.

[20] G. Chen and L. Chacón, "A multi-dimensional, energy- and charge-conserving, nonlinearly implicit, electromagnetic vlasov-darwin particle-in-cell algorithm," Comput. Phys. Commun., vol. 197, pp. 7387, 2015.

[21] P. N. Brown and Y. Saad, "Hybrid Krylov methods for nonlinear systems of equations," SIAM J. Sci. Statist. Comput., vol. 11, no. 3, pp. 450-481, 1990.

[22] D. A. Knoll and D. E. Keyes, "Jacobian-free Newton-Krylov methods: a survey of approaches and applications," Journal of Computational Physics, vol. 193, no. 2, pp. 357-397, 2004.

[23] D. G. Anderson, "Iterative procedures for nonlinear integral equations," J. Assoc. Comput. Mach., vol. 12, p. $547,1965$.

[24] H. Walker and P. NI, "Anderson acceleration for fixed-point iterations," SIAM J. Numer. Anal., vol. 49, p. 1715, 2013.

[25] J. J. Duderstadt and W. R. Martin, Transport theory. Wiley, New York, 1979.

[26] G. C. Pomraning, The equations of radiation hydrodynamics. Pergamon Press Oxford, New York „, [1st ed.] ed., 1973.

[27] H. Park, D. A. Knoll, R. M. Rauenzahn, C. K. Newman, J. D. Densmore, and A. B. Wollaber, “An efficient and time accurate, moment-based scale-bridging algorithm for thermal radiative transfer problems," SIAM Journal on Scientific Computing, vol. 35, pp. S18-S41, 2013. 
[28] J. Willert and H. Park, "Using residual monte carlo to solve the high-order problem within momentbased accelerated thermal radiative transfer equations," Journal of Computational Physics, vol. 276, pp. 405-421, 2014.

[29] J. Willert, H. Park, and D. Knoll, “A comparison of acceleration methods for solving the neutron transport k-eigenvalue problem," Journal of Computational Physics, vol. 274, pp. 681 - 694, 2014.

[30] H. Park, D. A. Knoll, R. M. Rauenzahn, A. B. Wollaber, and R. B. Lowrie, “Moment-based acceleration of monte carlo solution for multifrequency thermal radiative transfer problems," Journal of Computational and Theoretical Transport, vol. 43, no. 1-7, pp. 314-335, 2014.

[31] J. Densmore, H. Park, A. Wollaber, R. Rauenzahn, and D. Knoll, “Monte carlo simulation methods in moment-based scale-bridging algorithms for thermal radiative-transfer problems," Journal of Computational Physics, vol. 284, no. 0, pp. 40 - 58, 2015.

[32] J. Willert, H. Park, and W. Taitano, "Using anderson acceleration to accelerate the convergence of neutron transport calculations with anisotropic scattering," Nuclear Science and Engineering, vol. 181, pp. 342-350, 2015.

[33] J. Willert, H. Park, and W. Taitano, "Applying nonlinear diffusion acceleration to the neutron transport k-eigenvalue problem with anisotropic scattering," Nuclear Science and Engineering, vol. 181, pp. 351-360, 2015.

[34] E. N. Aristova, V. Y. Gol'din, and A. Kolpakov, "Multidimensional calculations of radiation transport by nonlinear quasi-diffusion method," in Proceeding of ANS International Conference on Mathematics and Computation, Reactor Physics and Environmental Analysis in Nuclear Applications, pp. 27-30, 1999.

[35] E. N. Aristova, "Simulation of radiation transport in a channel based on the quasi-diffusion method," Transport Theory and Statistical Physics, vol. 37, no. 5-7, pp. 483-503, 2008.

[36] D. Anistratov, “Consistent spatial approximation of the low-order quasi-diffusion equation on coarse grids," Nuclear Science and Engineering, vol. 149, pp. 138-161, 2005.

[37] E. W. Larsen and J. Young, "A functional monte carlo method for k-eigenvalue problems," Nuclear Science and Engineering, vol. 159, pp. 107-126, 2008.

[38] D. Y. Anistratov and V. Y. Gol'din, "Multilevel quasidiffusion methods for solving multigroup neutron transport k-eigenvalue problems in one-dimensional slab geometry," Nuclear Science and Engineering, vol. 169, no. 2, pp. 111-132, 2011.

[39] W. A. Wieselquist, D. Y. Anistratov, and J. E. Morel, "A cell-local finite difference discretization of the low-order quasidiffusion equations for neutral particle transport on unstructured quadrilateral meshes," Journal of Computational Physics, vol. 273, pp. 343 - 357, 2014. 
[40] R. Smith and P. Gent, "Reference maunal for the Parallel Ocean Program (POP)," Tech. Rep. Los Alamos Technical Report LA-UR-02-2484, Los Alamos National Laboratory, 2002.

[41] D. Lee, T. J. Downar, and Y. Kim, "Convergence analysis of the nonlinear coarse-mesh finite difference method for one-dimensional fixed-source neutron diffusion problem," Nuclear Science and Engineering, vol. 147, pp. 127-147, 2004.

[42] K.-S. Kim and M. D. DeHart, "Unstructured partial- and net-current based coarse mesh finite difference acceleration applied to the extended step characteristics method in newt," Annals of Nuclear Energy, vol. 38, no. 2-3, pp. 527 - 534, 2011.

[43] E. Wolters, E. Larsen, and W. Martin, "Hybrid monte-carlo-cmfd methods for accelerating fission source convergence," Nuclear Science and Engineering, vol. 174, pp. 286-299, 2013.

[44] E. W. Larsen and B. W. Kelley, "The relationship between the coarse-mesh finite difference and the coarse-mesh diffusion synthetic acceleration methods," Nuclear Science and Engineering, vol. 178, pp. 1-15, 2014.

[45] S. Yuk and N. Cho, "Whole core transport solution with 2-d/1-d fusion kernel via p-cmfd acceleration and p-cmfd embedding of nonoverlapping local/global iterations," Nuclear Science and Engineering, vol. 181, pp. 1-16, 2015.

[46] J. J. Honrubia, "A synthetically accelerated scheme for radiative-transfer calculations," Journal of Quantitative Spectroscopy \& Radiative Transfer, vol. 49, no. 5, pp. 491-515, 1993.

[47] J. Morel, T. Wareing, and K. Smith, "A linear-discontinuous spatial difference scheme for Sn radiative transfer calculations," Journal of Computational Physics, vol. 128, pp. 445-462, 1996.

[48] G. L. Ramone, M. L. Adams, and P. F. Nowak, "A transport synthetic acceleration method for transport iterations," Nuclear science and engineering, vol. 125, no. 3, pp. 257-283, 1997.

[49] M. L. Adams and E. W. Larsen, "Fast iterative methods for discrete-ordinates particle transport calculations," Progress in Nuclear Energy, vol. 40, no. 1, pp. 3-159, 2002.

[50] J. S. Warsa, T. Wareing, and J. Morel, "Krylov iterative methods and the degraded effectiveness of diffusion synthetic acceleration for multidimensional sn calculations in problem with material discontinuities," Nuclear Science and Engineering, vol. 147, pp. 218-248, 2004.

[51] J. Willert, C. T. Kelley, D. A. Knoll, and H. Park, "A hybrid deterministic/monte carlo method for solving the k-eigenvalue problem with a comparison to analog monte carlo solutions," Journal of Computational and Theoretical Transport, vol. 43, no. 1-7, pp. 50-67, 2014.

[52] J. Fleck and J. Cummings, "Implicit Monte Carlo scheme for calculating time and frequency dependent nonlinear radiation transport," Journal of Computational Physics, vol. 8, no. 3, pp. 313-342, 1971. 
[53] N. Gentile, "Implicit Monte Carlo diffusion - An acceleration method for Monte Carlo timedependent radiative transfer simulations," Journal of Computational Physics, vol. 172, no. 2, pp. 543571, 2001.

[54] J. D. Densmore, T. J. Urbatsch, T. M. Evans, and M. W. Buksas, "A hybrid transport-diffusion method for Monte Carlo radiative-transfer simulations," Journal of Computational Physics, vol. 222, no. 2, pp. 485-503, 2007.

[55] P. Bhatnagar, E. Gross, and M. Krook, "A model for collision processes in gases. i. small amplitude processes in charged and neutral one-component systems," Phys. Rev. Let., vol. 94, no. 3, pp. 511-525, 1954.

[56] C. Kelly, Iterative methods for linear and nonlinear equations. Society for industrial and applied mathematics, 1995.

[57] G. Sod, "A survey of several finite difference methods for systems of nonlinear hyperbolic conservation laws," J. Comp. Phys., vol. 27, pp. 1-31, 1978.

[58] N. Brilliantov and T. Porschel, Kinetic theory of granular gases. Oxford University press, 2004.

[59] C. K. Birdsall and A. B. Langdon, Plasma Physics via Computer Simulation. New York: McGraw-Hill, 2005.

[60] R. W. Hockney and J. W. Eastwood, Computer Simulation Using Particles. Bristol, UK: Taylor \& Francis, Inc, 1988.

[61] G. Chen, L. Chacón, and D. C. Barnes, "An energy- and charge-conserving, implicit, electrostatic particle-in-cell algorithm," J. Comput. Phys., vol. 230, pp. 7018-7036, 2011.

[62] C. W. Nielson and H. R. Lewis, "Particle-code models in the nonradiative limit," Methods in Computational Physics, vol. 16, pp. 367-388, 1976.

[63] A. Friedman, A. B. Langdon, and B. I. Cohen, "A direct method for implicit particle-in-cell simulation," Comments on plasma physics and controlled fusion, vol. 6, no. 6, pp. 225 - 36, 1981.

[64] B. I. Cohen, A. B. Langdon, and A. Friedman, "Implicit time integration for plasma simulation," J. Comput. Phys., vol. 46, no. 1, pp. $15-38,1982$.

[65] A. B. Langdon, B. I. Cohen, and A. Friedman, "Direct implicit large time-step particle simulation of plasmas," J. Comput. Phys., vol. 51, no. 1, pp. $107-38,1983$.

[66] D. C. Barnes, T. Kamimura, J. N. Leboeuf, and T. Tajima, "Implicit particle simulation of magnetized plasmas," J. Comput. Phys., vol. 52, no. 3, pp. 480 - 502, 1983. 
[67] A. B. Langdon and D. C. Barnes, "Direct implicit plasma simulation," in Multiple time scales (J. U. Brackbill and B. I. Cohen, eds.), pp. 335-375, Academic Press, New York, 1985.

[68] B. I. Cohen, "Multiple time-scale methods in particle simulations of plasma," Particle Accelerators, vol. 19, pp. 227-236, 1986.

[69] R. J. Mason, "An electromagnetic field algorithm for 2d implicit plasma simulation," Journal of Computational Physics, vol. 71, no. 2, pp. 429-473, 1987.

[70] D. W. Hewett and A. B. Langdon, "Electromagnetic direct implicit plasma simulation," J. Comput. Phys., vol. 72, no. 1, pp. $121-55,1987$.

[71] A. Friedman, "A second-order implicit particle mover with adjustable damping," J. Comput. Phys., vol. 90, no. 2, pp. 292-312, 1990.

[72] T. Kamimura, E. Montalvo, D. C. Barnes, J. N. Leboeuf, and T. Tajima, "Implicit particle simulation of electromagnetic plasma phenomena," J. Comput. Phys., vol. 100, no. 1, pp. 77-90, 1992.

[73] M. Gibbons and D. Hewett, "The Darwin Direct Implicit Particle-in-Cell (DADIPIC) method for simulation of low frequency plasma phenomena," J. Comput. Phys., vol. 120, pp. 231-247, 1995.

[74] B. I. Cohen, A. B. Langdon, D. W. Hewett, and R. J. Procassini, "Performance and optimization of direct implicit particle simulation," J. Comput. Phys., vol. 81, no. 1, pp. 151 - 168, 1989.

[75] S. Markidis and G. Lapenta, "The energy conserving particle-in-cell method," Journal of Computational Physics, vol. 230, no. 18, pp. 7037-7052, 2011.

[76] G. Lapenta and S. Markidis, "Particle acceleration and energy conservation in particle in cell simulations," Physics of Plasmas, vol. 18, p. 072101, 2011.

[77] D. W. Hewett, "Elimination of electromagnetic radiation in plasma simulation: The Darwin or magnetoinductive approximation," Space Science Reviews, vol. 42, pp. 29-40, 1985.

[78] P. Degond and P.-A. Raviart, "An analysis of the Darwin model of approximation to Maxwell's equations," Forum Math, vol. 4, no. 4, pp. 13-44, 1992.

[79] P.-A. Raviart and E. Sonnendrücker, "A hierarchy of approximate models for the Maxwell equations," Numerische Mathematik, vol. 73, no. 3, pp. 329-372, 1996.

[80] T. B. Krause, A. Apte, and P. Morrison, "A unified approach to the Darwin approximation," Physics of Plasmas, vol. 14, p. 102112, 2007.

[81] G. Chen and L. Chacón, "An analytical particle mover for the charge-and energy-conserving, nonlinearly implicit, electrostatic particle-in-cell algorithm," Journal of Computational Physics, vol. 247, pp. 79-87, 2013. 
[82] L. Chacón, G. Chen, and D. C. Barnes, "A charge- and energy-conserving implicit, electrostatic particle-in-cell algorithm on mapped computational meshes," J. Comput. Phys., vol. 233, pp. 1-9, 2013.

[83] L. Chacón and G. Chen, "A curvilinear, fully implicit, conservative electromagnetic PIC algorithm in multiple dimensions," Journal of computational physics, 2016.

[84] A. B. Langdon, "Analysis of the time integration in plasma simulation," J. Comput. Phys., vol. 30, no. 2, pp. $202-21,1979$.

[85] R. Kingham and A. Bell, "Nonlocal magnetic-field generation in plasmas without density gradients," Phys. Rev. Lett., vol. 88, p. 045004, 2002.

[86] R. Kingham and A. Bell, "An implicit Vlasov-Fokker-Planck code to model non-local electron transport in 2-d with magnetic fields," J. Comput. Phys., vol. 194, pp. 1-34, 2004.

[87] C. Ridgers, R. Kingham, and A. Thomas, "Magnetic cavitation and the reemergence of nonlocal transport in laser plasmas," Phys. Rev. Lett., vol. 100, p. 075003, 2008.

[88] T. Kho and M. Haines, "Nonlinear kinetic transport of electrons and magnetic field in laser-produced plasmas," Phys. Rev. Lett., vol. 55, pp. 825-828, 1985.

[89] J. Luciani, P. Mora, and A. Bendib, "Magnetic field and nonlocal transport in laser-created plasmas," Phys. Rev. Lett., vol. 55, pp. 2421-2424, 1985.

[90] E. Epperlein, G. Rickard, and A. Bell, "A code for the solution of the Vlasov-Fokker-Planck equation in 1-D and 2-D," Comp. Phys. Comm., vol. 52, pp. 7-13, 1988.

[91] R. Town, A. Bell, and A. Bell, "Fokker-Planck simulations of short-pulse-laser-solid experiments," Rhys. Rev. E., vol. 50, no. 2, part B, pp. 1413-1421, 1994.

[92] E. Epperlein, “Implicit and conservative difference scheme for the Fokker-Planck equation," J. Comp. Phys., vol. 112, pp. 291-297, 1994.

[93] L. Chacón, D. Barnes, D. Knoll, and G. Miley, “An implicit energy conservatie 2D Fokker-Planck algorithm I. difference scheme," J. Comp. Phys., vol. 157, pp. 618-653, 2000.

[94] L. Chacón, D. C. Barnes, D. A. Knoll, and G. H. Miley, “An implicit energy conservative 2D FokkerPlanck algorithm II. Jacobian-free Newton-Krylov solver," J. Comp. Phys., vol. 157, pp. 654-682, 2000.

[95] V. Mousseau and D. Knoll, "Fully implicit kinetic solution of collisional plasmas," J. Comp. Phys., vol. 136, pp. 308-323, 1997.

[96] L. Gardner, G. Gardner, and S. Zaki, “Collisional effects in plasmas modeled by a simplified FokkerPlanck equation," J. Comput. Phys., vol. 107, pp. 40-50, 1993. 
[97] A. Lenard and I. Bernstein, "Plasma oscillations with diffusion in velocity space," Phys. Rev. Lett., vol. 112, pp. 1456-1459, 1958.

[98] A. Thomas, R. Kingham, and C. Ridgers, "Rapid self-magnetization of laser speckles in plasmas by nonlinear anisotropic instability," New J. Phys., vol. 11, p. 033001, 2009.

[99] T. Johnston, “Cartesian tensor scalar product and spherical harmonic expansions in Boltzmann's equation," Phys. Rev. Lett., vol. 120, pp. 1103-1111, 1960.

[100] A. Thomas, M. Tzoufras, A. Robinson, R. Kingham, and C. Ridgers, "A review of Vlasov-FokkerPlanck numerical modeling of inertial confinement fusion plasma," J. Comput. Phys., vol. 231, pp. 1051-1079, 2012.

[101] W. Taitano and L. Chacón, “Charge-and-energy conserving moment-based accelerator for multispecies Vlasov-Fokker-Planck-Ampère system, part I: collisionless aspects," J. Comput. Phys., vol. 284, pp. 718-736, 2015.

[102] W. Taitano, D. Knoll, and L. Chacón, "Charge-and-energy conserving moment-based accelerator for multi-species Vlasov-Fokker-Planck-Ampère system, part II: collisional aspects," J. Comput. Phys., vol. 284, pp. 737-757, 2015.

[103] W. Taitano, L. Chacón, A. Simakov, and K. Molvig, "A mass, momentum, and energy conserving, fully implicit, scalable algorithm for the multi-dimensional, multi-species Rosenbluth-Fokker-Planck equation," J. Comput. Phys., vol. 297, pp. 357-380, 2015.

[104] W. Taitano, L. Chacón, and A. Simakov, “An adaptive, conservative 0D-2V multispecies RosenbluthFokker-Planck solver for arbitrarily disparate mass and temperature regimes," J. Comput. Phys., vol. 318, pp. 391-420, 2016.

[105] M. Rosenbluth, W. MacDonald, and D. Judd, "Fokker-Planck equation for an inverse-square force," Phys. Rev. Lett., vol. 107, no. 1, pp. 1-6, 1956.

[106] C. Cheng and G. Knorr, "The integration of the Vlasov equation in configuration space," J. Comput. Phys., vol. 22, pp. 330-351, 1976.

[107] F. Filbet, E. Sonnendrócker, and P. Bertrand, "Conservative numerical scheme for the Vlasov equation," J. Comput. Phys., vol. 172, pp. 176-187, 2001.

[108] J. Willert, W. Taitano, and D. Knoll, "Leveraging Anderson acceleration for improved convergence of iterative solutions to transport systems," J. Comp. Phys., vol. 273, pp. 278-286, 2014.

[109] S. Braginskii, "Transport processes in a plasmas," in Review of Plasma Physics (M. Leontovich, ed.), Consultants Bereau, NY, 1982. 
[110] M. Maltrud and J. McClean, "An eddy resolving global 1/10 ocean simulation," Ocean Model., vol. 8, no. 1, pp. 31-54, 2005.

[111] R. Hallberg, "Stable split time stepping schemes for large-scale ocean modeling," J. Comput. Phys., vol. 135, no. 1, pp. 54-65, 1997.

[112] R. Higdon and R. de Szoeke, "Barotropic-baroclinic time splitting for ocean circulation modeling," J. Comput. Phys., vol. 135, no. 1, pp. 30-53, 1997.

[113] T. Ringler, M. Petersen, R. Higdon, D. Jacobsen, P. Jones, and M. Maltrud, "A multi-resolution approach to global ocean modeling," Ocean Model., vol. 69, pp. 211-232, 2013.

[114] R. Pacanowski, K. Dixon, and A. Rosati, “The GFDL Modular Ocean Model Users Guide,” Tech. Rep. 2, Geophysical Fluid Dynamics Laboratory, Princeton, USA, 1993.

[115] C. Newman, G. Womeldorff, L. Chacón, and D. A. Knoll, “High-order/low-order methods for ocean modeling," Procedia Computer Science, vol. 51, pp. 2086-2096, 2015.

[116] C. Newman, G. Womeldorff, D. A. Knoll, and L. Chacón, “A communication-avoiding implicitexplicit method for a free-surface ocean model," J. Comput. Phys., vol. 305, pp. 877-894, 2016.

[117] U. M. Ascher, S. J. Ruuth, and B. T. Wetton, "Implicit-explicit methods for time-dependent partial differential equations," SIAM J. Numer. Anal., vol. 32, no. 3, pp. 797-823, 1995.

[118] D. R. Durran and P. N. Blossey, "Implicit-explicit multistep methods for fast-wave-slow-wave problems," Mon. Weather Rev., vol. 140, no. 4, pp. 1307-1325, 2012.

[119] J. Kim and P. Moin, “Application of a fractional-step method to incompressible navier-stokes equations," J. Comput. Phys., vol. 59, no. 2, pp. 308-323, 1985.

[120] J. Lambert, Numerical methods for ordinary differential systems: the initial value problem. John Wiley \& Sons, Inc., 1991.

[121] C. Newman and D. A. Knoll, "Physics-based preconditioners for ocean simulation," SIAM J. Sci. Comput., vol. 35, no. 5, pp. S445-S464, 2013.

[122] A. Arakawa and V. Lamb, "Computational Design of the Basic Dynamical Processes of the UCLA General Circulation Model," in Methods in Computational Physics (J. Chang, ed.), vol. 17, pp. 173-265, Academic Press, New York, 1977. 